\title{
Poloidal trapping of the high-frequency Alfvén continuum and eigenmodes in stellarators
}

\author{
Yu. V. Yakovenko', A. Weller ${ }^{2}$, A. Werner ${ }^{2}$, S. Zegenhagen ${ }^{2}$, \\ O. P. Fesenyuk ${ }^{1}$, Ya. I. Kolesnichenko ${ }^{1}$ \\ ${ }^{1}$ Institute for Nuclear Research, Prospekt Nauky 47, Kyiv 03680, Ukraine \\ ${ }^{2}$ Max-Planck-Institut für Plasmaphysik, IPP-EURATOM Association, D-17489 \\ Greifswald, Germany
}

\begin{abstract}
Alfvén oscillations in three-dimensional toroidal magnetic configurations (stellarators) are considered. It is shown that the wave functions of the Alfvén continuum can be trapped in certain "waveguides" when there are sufficiently large harmonics with sufficiently close periods along the magnetic field lines in the Fourier spectrum of the magnetic configuration. Such trapping is typical in the frequency range of the helicity-induced continuum gaps, in which case the wave functions are typically trapped at the inner circumference of the magnetic flux surface. Trapping also takes place near the crossings of continuum gaps, the localization of the wave becoming stronger with approach to the crossing point. At the crossing point, the continuum wave functions are localized at single field lines, which is shown to result in "annihilation" of the gaps (the width of the joint gap is the difference of the widths that the two gaps would have is they were alone). It is shown with the use of the ballooning formalism that the Alfvén eigenmodes (AEs) associated with the trapped continua are also trapped in regions of the same shape. Experimental observations on the stellarator Wendelstein 7-AS are reported, which indicate that trapping of AEs at the inner circumference of the plasma indeed takes place.
\end{abstract}

\section{Introduction}

Spectra of the shear Alfvén waves attract considerable attention due to the ability of oscillations of the discrete spectrum (Alfvén eigenmodes, AE) to be destabilized by fast ions. Alfvén instabilities have been observed in many experiments on tokamaks and stellarators $[1,2,3]$. They are of interest since they can lead to enhanced loss of fast ions $[1,2,3]$ and considerably affect the confinement of the thermal plasma [2, 4]. In addition, such instabilities can be used for diagnostics (MHD spectroscopy [5, 6]) and may offer a possibility to remove helium ash from a stellarator reactor [7].

Historically, the investigation of Alfvén spectra in non-uniform plasmas was first carried out for the one-dimensional (slab or cylindrical) geometry of the magnetic configuration. It was found that the variation of the the Alfvén velocity, $v_{A}$, and the longitudinal wave number, $k_{\|}$, with the radial coordinate, $r$, produces the continuous spectrum at the frequencies $\omega=k_{\|}(r) v_{A}(r)$. Shear Alfvén oscillations with the frequency 
in the continuum are damped near the flux surface where the local Alfvén resonance $\omega=k_{\|}(r) v_{A}(r)$ occurs [8]. Outside the continuum there may exist AEs - Global Alfvén Eigenmodes (GAE) [9, 10], which are weakly damped.

The two-dimensionality (poloidal asymmetry) of a toroidal magnetic configuration produces frequency gaps in the Alfvén continuum [11], which makes possible the existence of other types of AEs - the Toroidicity-induced Alfvén Eigenmodes (TAE [12]), the Ellipticity-induced Alfvén Eigenmodes (EAE [13]) and the Noncircular triangularityinduced Alfvén Eigenmodes (NAE [13]). Non-ideal effects (the finite Larmor radius and the electron inertia) are responsible for the existence of kinetic counterparts of the gap eigenmodes - kinetic TAEs (KTAE [14]) and kinetic EAEs (KEAE [15]). In addition, several types of Energetic-Particle Modes (EPM), i.e., the modes that are not only destabilized by energetic ions but owe their existence to them, are possible [16].

The absence of the toroidal symmetry in a stellarator complicates the Alfvén spectrum, resulting in the appearance of new gaps in the high-frequency part of the spectrum. These gaps may contain AEs of new types - Helicity-induced and Mirrorinduced Alfvén Eigenmodes (HAE and MAE) [17, 18, 19, 20]. However, the effect of the three-dimensionality of the stellarator geometry on the Alfvén wave spectra is not restricted to a simple multiplication of the continuum gaps. It has been found that the three-dimensionality qualitatively changes the structure of the AC. First of all, the coefficients of the equation for the Alfvén waves in a three-dimensional (3D) configuration are quasiperiodic rather than periodic along the magnetic field lines (at the flux surfaces where the rotational transform, $\iota$, is irrational). As a result, the AC has the structure of a Cantor set at the irrational- $\iota$ flux surfaces, and the AC equation can possess not only continuous but also dense discrete spectra [21]. Recently, it was found that the interference of two Fourier harmonics of the equilibrium quantities with close periods along the magnetic field can result in new phenomena ("annihilation" of the AC gaps at their crossing points [22]; trapping of Alfvén waves in certain angular sectors (waveguides) $[23,24,25]$ ).

In this work, we study the properties of the $\mathrm{AC}$ and $\mathrm{AEs}$ in a frequency vicinity of two continuum gaps produced by equilibrium harmonics with close periods along the magnetic field (note that this means that the gaps are close in frequency). Some preliminary results on this topic were reported at conferences [22, 23, 24, 25] and included to an invited paper [25], but most of the material of the present paper has never been published in journal papers. The limit case when the two harmonics have equal periods along the field lines at a certain flux surface is considered (which means that the corresponding gaps cross at this flux surface; note that this is possible only in $3 \mathrm{D}$ configurations). We show that in this case the wave functions of the $\mathrm{AC}$ are localized at single field lines, which leads to the "annihilation" of the gaps: the width of the joint gap is equal to the difference of the widths that the gaps would have if they were alone. Considering the case when the gaps are at a sufficiently small but finite distance, we find that the $\mathrm{AC}$ wave functions are trapped in certain regions ("waveguides") of the flux surfaces, which drastically changes the structure of the continuum. We demonstrate that 
continuum gaps in the high-frequency part of the AC in many stellarators are typically sufficiently close in frequency for the waves to be trapped. We use the ballooning formalism [26] to show that the AEs in the corresponding frequency range are also trapped in the waveguides of the same structure. Finally, we present experimental observations of Alfvénic activity in the stellarator Wendelstein 7-AS (W7-AS [27]), which indicate that some AEs are trapped at the inner circumference of the plasma torus in agreement with theory predictions.

The structure of the work is as follows. In section 2, the $\mathrm{AC}$ in the vicinity of two close gaps is investigated. In particular, in subsection 2.1 basic properties of the AC are introduced, and peculiarities of the high-frequency part of the AC in stellarators are discussed. In subsection 2.2, the structure of the $\mathrm{AC}$ at a point where the two gaps cross is elucidated, and the phenomenon of the gap annihilation is demonstrated. Results of a numerical study of the AC near the crossing point are presented in subsection 2.3. In subsection 2.3, an analytical explanation of the numerical results is given. In section 3, the phenomenon of the localization of AEs produced by two interacting gaps in certain waveguides is demonstrated. An experimental observation of poloidally localized Alfvén instabilities is presented in section 4. Finally, the conclusions are presented in section 5.

\section{Continuum in the vicinity of two close continuum gaps}

\subsection{Basic properties of the continuum}

Assuming that the toroidal plasma under consideration possesses nested magnetic flux surfaces, we use Boozer magnetic coordinates [28] $\left(x^{\psi}, x^{\theta}, x^{\phi}\right)=(\psi, \theta, \phi)$ with $\psi$ the toroidal magnetic flux, $\theta$ and $\phi$ the poloidal and toroidal angles, respectively. Then the continuous spectrum of the ideal Alfvén oscillations is described by the following equation $[19,29]$ :

$$
\hat{L}\left(h_{g}^{\psi \psi} \hat{L} \Phi\right)+\omega^{2} \frac{R^{2}}{\left\langle v_{A}\right\rangle^{2}} h_{c}^{\psi \psi} \Phi=0,
$$

where $\omega$ is the frequency; $\Phi(\psi, \theta, \phi)$ is the wave function (the scalar potential of the electromagnetic field perturbation); $\hat{L}=\partial / \partial \phi+\iota(\psi) \partial / \partial \theta$ is an operator of differentiation along the field lines; $h_{c}^{\psi \psi}=h_{g}^{\psi \psi} / h_{B}^{4} ; h_{g}^{\psi \psi}(\psi, \theta, \phi)=g^{\psi \psi} /\left\langle g^{\psi \psi}\right\rangle ;\langle\ldots\rangle$ denotes flux surface averaging; $g^{\psi \psi}=|\nabla \psi|^{2}$ is the corresponding component of the contravariant metric tensor; $h_{B}(\psi, \theta, \phi)=B /\langle B\rangle ; B$ is the magnetic field strength; $v_{A}=B /(4 \pi \rho)^{1 / 2}$ is the Alfvén velocity; $\rho=\rho(\psi)$ is the plasma mass density; $R(\psi)=\langle B\rangle \sqrt{g} h_{B}^{2}$ is approximately the major radius of the plasma torus; $g$ is the metric tensor determinant. Equation (1) includes only derivatives by angles with $\psi$ playing the part of a parameter. Supplemented with natural boundary conditions of periodicity in $\theta$ and $\phi$, this equation determines a set of eigenfrequencies, which form the branches of the continuum as $\psi$ is varied. In fact, the continuum equation (1) is a generalization of the Alfvén wave dispersion relation, $\omega=k_{\|} v_{A}$, to inhomogeneous plasmas with complicated geometry (setting $h_{g}^{\psi \psi}=h_{c}^{\psi \psi}=1$ and $\hat{L}=i k_{\|}$in the 
former, we obtain the latter). However, as mentioned above, the physical meaning of this dispersion relation in inhomogeneous plasmas is different. Instead of determining the frequencies at which the wave can propagate, it determines the frequencies of the local resonances of the Alfvén wave, perturbations with the resonance frequencies being intensively absorbed at the resonant flux surfaces [8].

Actually, the AC equation (1) includes only derivatives along a field line. Written in the coordinates $(\psi, \alpha \equiv \theta-\iota \phi, \phi)$, where $\alpha$ is a field line label, it becomes an ordinary differential equation:

$$
\frac{\mathrm{d}}{\mathrm{d} \phi}\left(h_{g}^{\psi \psi} \frac{\mathrm{d} \hat{\Phi}}{\mathrm{d} \phi}\right)+\Omega^{2} h_{c}^{\psi \psi} \hat{\Phi}=0,
$$

where $\Omega=\omega R /\left\langle v_{A}\right\rangle$ is the dimensionless frequency, and $\alpha$ is an additional parameter. The price of this simplification is that the wave function $\Phi$ and the coefficients of the equation, generally speaking, lose their periodicity: When $\iota$ is irrational, a function periodic in $\theta$ and $\phi$ is quasiperiodic on a single field line. The simplest way to set the boundary conditions is then to require that $\Phi$ is finite at $\phi= \pm \infty$. However, then we do not observe the periodicity properties of $\Phi$. As we will see, this may change the spectrum of the equation for rational $\iota$.

It will be convenient to have the $\mathrm{AC}$ equation presented also in a matrix from. With this aim, we expand $h_{B}, h_{g}^{\psi \psi}$, and $h_{c}^{\psi \psi}$ in Fourier series:

$$
\begin{aligned}
& h_{B}=1+\frac{1}{2} \sum_{\mu, \nu} \epsilon_{B(\mu, \nu)} \exp (i \mu \theta-i \nu N \phi), \\
& h_{g, c}^{\psi \psi}=1+\frac{1}{2} \sum_{\mu, \nu} \epsilon_{g, c(\mu, \nu)}^{\psi \psi} \exp (i \mu \theta-i \nu N \phi),
\end{aligned}
$$

where $N$ is the number of the configuration periods. We will refer to the Fourier coefficients $\epsilon_{B(\mu, \nu)}$ and $\epsilon_{g, c(\mu, \nu)}^{\psi \psi}$ will be as coupling parameters (since they characterize the coupling of different harmonics of the wave) and to $\mu$ and $\nu$ as the poloidal and toroidal coupling numbers, respectively. Taking into account which possible couplings between harmonics of $\Phi$ can result from equations (3) and (4), we take the wave function at a single field line $(\theta=\alpha+\iota \phi)$ in the form

$$
\Phi=\exp (i \tilde{k} \phi) \sum_{p, s=-\infty}^{\infty} \Phi_{p s} \exp [i(p \iota-i s N) \phi+i p \alpha] .
$$

where $\tilde{k}=k_{\|} R$ is the normalized longitudinal wave number of the leading harmonic of the wave. Then equation (2) takes the matrix form

$$
\sum_{p, s=-\infty}^{\infty} \mathcal{G}_{p_{*}, s_{*} ; p, s}(\psi, \tilde{k}) \Phi_{p s}=\Omega^{2} \sum_{p, s=-\infty}^{\infty} \mathcal{C}_{p_{*}, s_{*} ; p, s}(\psi) \Phi_{p s}
$$

where $\mathcal{G}$ and $\mathcal{C}$ are infinite Hermitian matrices given by

$$
\mathcal{G}_{p_{*}, s_{*} ; p, s}(\tilde{k})=\left(\delta_{p_{*}, p} \delta_{s_{*}, s}+\frac{1}{2} \epsilon_{g\left(p_{*}-p, s_{*}-s\right)}^{\psi \psi}\right) \tilde{k}_{\left(p_{*} s_{*}\right)} \tilde{k}_{(p s)},
$$




$$
\mathcal{C}_{p_{*}, s_{*} ; p, s}=\delta_{p_{*}, p} \delta_{s_{*}, s}+\frac{1}{2} \epsilon_{c\left(p_{*}-p, s_{*}-s\right)}^{\psi \psi}
$$

$\tilde{k}_{(p s)}=\tilde{k}+p \iota-s N$ are the normalized longitudinal wave number of the corresponding harmonic of $\Phi, \delta_{i, j}$ is the Kronecker symbol. The matrix form shows that the spectrum of equation (2), in fact, does not depend on the field line (the parameter $\alpha$ does not enter equation (6)). If we restore the constraint that $\Phi$ is periodic in $\theta$ and $\phi$ then equation (5) should be compatible with a standard Fourier representation of $\Phi$, which is possible when $\tilde{k}=m \iota-n$ for some integer $m$ and $n$. When $\iota$ is irrational, any real number can be approximated by $m \iota-n$ with arbitrary accuracy for some $m$ and $n$. At the same time, if $\iota$ is not constant, for any flux surface with rational value of $\iota$ there are surfaces with irrational $\iota$ at arbitrarily close distance. For this reason, when we are interested in the AC in general rather than in the behaviour of its separate branches, we do not need to care that $\tilde{k}=m \iota-n$.

In the cylindrical geometry, when different Fourier harmonics of the wave are decoupled, the AC consists of the corresponding branches $\Omega=\Omega_{m, n}(\psi)=\left|\tilde{k}_{m, n}\right|$, where $\tilde{k}_{m, n}=m \iota-n$ is the normalized longitudinal wave number of the harmonic $(m, n)$, $m$ and $n$ are the poloidal and toroidal wave numbers, respectively. To see this, it is sufficient to take $\Phi \propto \exp (i m \theta-i n \phi)$ in equation (2) or omit all non-diagonal elements in equation (6).

Deviations from the cylindrical geometry lead to coupling of different wave harmonics. When the coupling is weak, we can study only interactions of pairs of the harmonics. Let us consider a pair of wave harmonics, $(m, n)$ and $(m+\mu, n+\nu N)$, interacting via the Fourier harmonic $(\mu, \nu)$ of the magnetic configuration, i.e., the harmonic $\propto \exp (i \mu \theta-i \nu N \phi)$. Taking the corresponding $2 \times 2$ sub-matrix of equation (6), one can see that the smaller is the difference $\left|\tilde{k}_{m, n}\right|-\left|\tilde{k}_{m+\mu, n+\nu N}\right|$, the stronger is the interaction. The consequences of the interaction are most serious when $\tilde{k}_{m, n}=$ $-\tilde{k}_{m+\mu, n+\nu N}= \pm \tilde{k}_{*(\mu, \nu)}$, where $\tilde{k}_{*(\mu, \nu)}=(\mu \iota-\nu N) / 2$ is the normalized characteristic longitudinal wave number associated with the $(\mu, \nu)$ coupling. This condition determines the line

$$
\Omega_{*(\mu, \nu)}(\psi) \approx\left|\tilde{k}_{*(\mu, \nu)}\right|
$$

on the plane $(\psi, \Omega)$ (this line is the locus of the crossings of pairs of cylindrical continuum branches $\Omega=\Omega_{m, n}(\psi)$ and $\Omega=\Omega_{m+\mu, n+\nu N}(\psi)$ for arbitrary $m$ and $\left.n\right)$. Near this line, the $(\mu, \nu)$ coupling (i.e., the coupling via the $(\mu, \nu)$ harmonic) produces a gap in the continuum, which we will label with the numbers $(\mu, \nu)$. The width of this gap is about [19]

$$
\delta \Omega / \Omega \approx\left|\epsilon_{(\mu, \nu)}^{\psi \psi}\right|
$$

where $\epsilon_{(\mu, \nu)}^{\psi \psi}=\left(\epsilon_{g(\mu, \nu)}^{\psi \psi}+\epsilon_{c(\mu, \nu)}^{\psi \psi}\right) / 2 \approx \epsilon_{g(\mu, \nu)}^{\psi \psi}-2 \epsilon_{B(\mu, \nu)}$.

When the coupling is strong, which is typically the case in stellarators because of strong shaping, the width and position of the gaps cannot be found by considering only interactions of pairs of wave harmonics. The width of the $(\mu, \nu)$ gap is contributed not 
only by the $(\mu, \nu)$ harmonics of the coefficients but also by all possible combinations of harmonics $\left(\mu_{1}, \nu_{1}\right), \ldots,\left(\mu_{k}, \nu_{k}\right)$ such that $\left(\mu_{1}, \nu_{1}\right)+\ldots+\left(\mu_{k}, \nu_{k}\right)=(\mu, \nu)$. As a result, the gap with certain $(\mu, \nu)$ may appear even when the direct $(\mu, \nu)$ coupling is absent or negligible (we will refer to such gaps as "combination" gaps). In addition, numerical calculations show that the interaction between the gaps displaces them, so that their frequencies may sometimes differ from that given by equation (9) by a factor of two or even more [19]. Then a natural question appears: Is there a way to label the gaps in this case, i.e., to attribute the corresponding coupling numbers $(\mu, \nu)$ to each gap?

This problem has already been addressed in the theory of the Schrödinger equation with a quasiperiodic potential [30] and in the paper [21]. The substitution $\Phi=$ $\left(h_{g}^{\psi \psi}\right)^{-1 / 2} \tilde{\Phi}$ transforms equation (2) into an equation very similar to the Schrödinger equation (the only difference is in the way the eigenvalue enters the equation, which does not seem to be essential). The potential of this equation, like the coefficients of equation (2), is quasiperiodic for irrational $\iota$. Theory [30] states that a "natural" labelling for a Schrödinger equation can be found from the so-called "winding number", which shows how many times per unit length the eigenfunctions of the equation at the boundaries of the spectral gap oscillate around zero.

Another method to label the AC gaps, which is more convenient when the problem is treated in the matrix formulation, is used in the AC code COBRA [19]. Equation (6) is solved with reduced non-diagonal elements of the matrices $\mathcal{C}$ and $\mathcal{G}$ (they are multiplied by a small factor) and $\tilde{k}=-\tilde{k}_{*(\mu, \nu)} / 2$. It is then assumed that the boundaries of the gap $(\mu, \nu)$ are determined by those eigenfrequencies of (6) which correspond to the eigenvectors with the prevailing contribution of the harmonics with $(p, s)=(0,0)$ and $(\mu, \nu)$. Since $-\tilde{k}_{(0,0)}=\tilde{k}_{(\mu, \nu)}=\tilde{k}_{*(\mu, \nu)} / 2$, one can show that the winding number of these harmonics has exactly the magnitude required by the theory [30] for the boundaries of the gap $(\mu, \nu)$. Then the code gradually increases the non-diagonal elements until they reach their actual magnitudes and follows the continuations of the two selected eigensolutions. It is assumed that on the end of this process the boundaries of the gap are given by the eigenfrequencies of the two selected continuum branches. Our experience of calculations has shown that this method gives reasonable results. It seems to be equivalent to the winding-number method, although we have not tried to prove this fact rigorously (in fact, it is sufficient to prove that the winding number is a continuous function of the coupling parameters).

As an example, let us consider the AC calculated with COBRA for W7-AS shot No. 56936 (figure 1). One can see that the high-frequency part of the AC is in striking contrast to the low-frequency one: in the low-frequency part (up to $150 \mathrm{kHz}$ ), the gaps separate rather wide continuum bands, whereas in the high-frequency part (in the range of helicity-induced and mirror-induced gaps) the continuum is compressed by wide gaps into extremely thin threads. For instance, the relative width of the continuum wall between the $(2,1)$ and $(3,1)$ gaps at $r / a=0.3$ is as small as $\delta \omega / \omega \leq 6 \times 10^{-4}$. If these two gaps were narrow, the relative distance between them could be estimated 


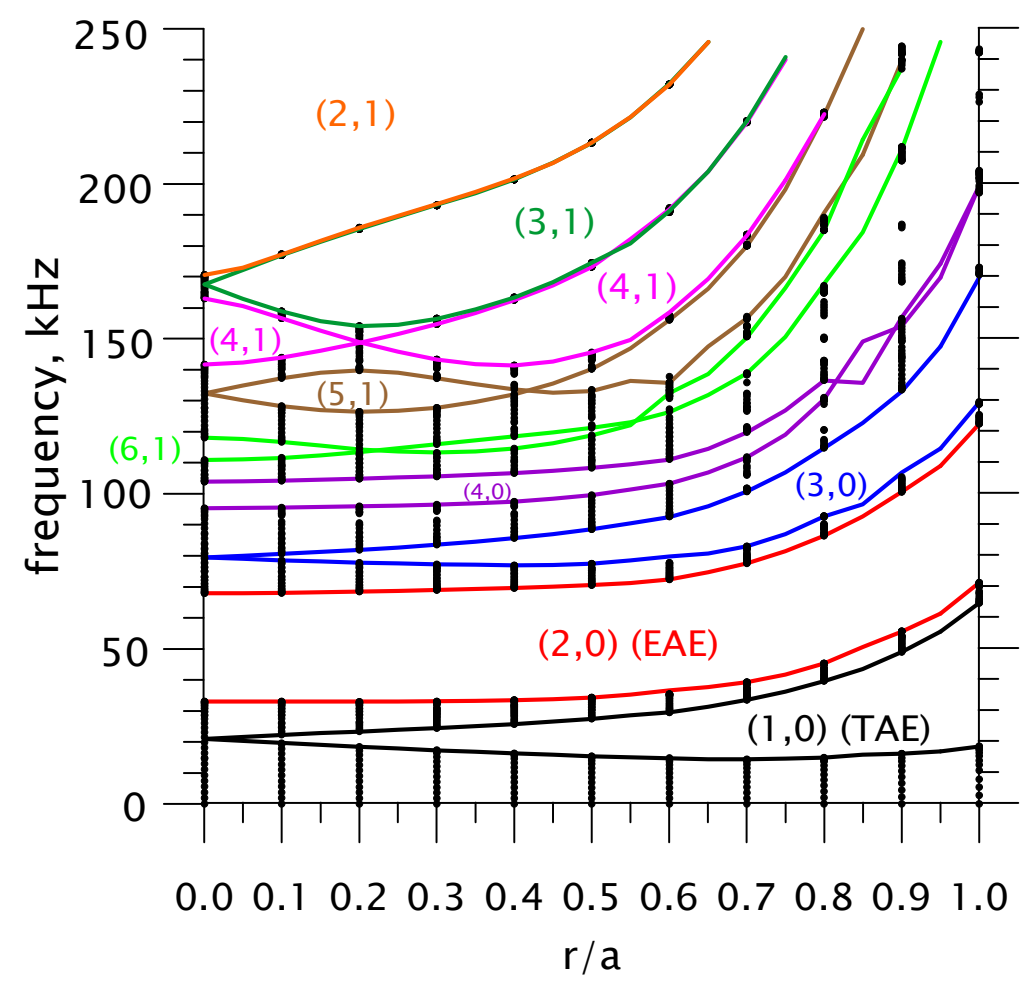

Figure 1. Alfvén continuum in W7-AS shot No. 56936.

from equation (9) as

$$
2 \frac{\Omega_{*(2,1)}-\Omega_{*(3,1)}}{\Omega_{*(2,1)}+\Omega_{*(3,1)}}=2 \frac{\left|\tilde{k}_{*(2,1)}-\tilde{k}_{*(3,1)}\right|}{\left|\tilde{k}_{*(2,1)}+\tilde{k}_{*(3,1)}\right|}=\frac{\iota}{N-2.5 \iota} \approx 0.12 .
$$

at $r=0.3 a$. At the same time, the relevant coupling parameters are

$$
\epsilon_{(2,1)}^{\psi \psi}=0.59, \quad \epsilon_{(3,1)}^{\psi \psi}=0.35 .
$$

Due to equation (10), these parameters are estimates of the relative widths of these gaps (of course, these estimates are valid only for narrow gaps; the actual widths of the gaps are different). We observe that the estimated gap widths well exceed the estimated distance between them (the gaps have "not enough space"). Then it seems understandable why the continuum wall between the gaps is very narrow. However, it is not immediately clear how as small number as $6 \times 10^{-4}$ can be obtained from these three numbers. The wave functions with the frequencies in this continuum wall possess complicated Fourier spectra, the wave almost vanishing at the outer circumference of the torus ("anti-ballooning" behaviour). An example is the wave function $(m, n)=(0,2)$ with the frequency in the continuum thread between the $(2,1)$ and $(3,1)$ gaps at $r / a=0.3$ (figure 2).

The AC in LHD (Large Helical Device [31]) exhibits the same general pattern (see figure 7 in [32]): The continua in the high frequency range look like thin threads separated by wide gaps. One can suppose that the reason for this is the same as in W7AS. The estimated relative distance between the helicity-induced gaps is $\sim \iota / N$, which 


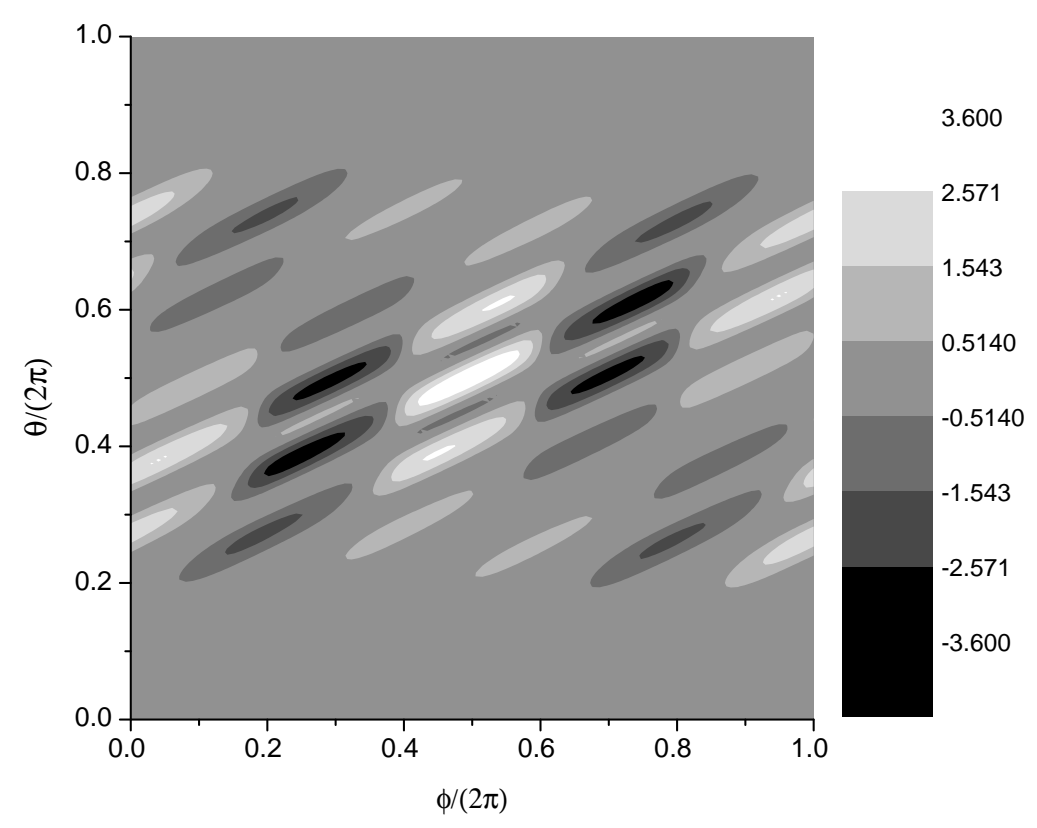

Figure 2. Wave function of the continuum branch $(m, n)=(0,2)$ at $r / a=0.3$ in W7-AS shot No. 56936.

is less than the characteristic magnitude of the coupling parameters associated with helicity. It seems that this is typical, at least, for high- $N$ stellarators, which motivates us to study the structure of the AC and the AEs in this situation.

\subsection{Crossing of two gaps and gap annihilation}

Since we are interested in the case when the distance between the gaps is very small, it seems reasonable to consider first the limit case when the distance between two gaps totally vanishes, i.e., when they cross at a certain flux surface. Let us consider two gaps with the coupling numbers $\left(\mu_{1}, \nu_{1}\right)$ and $\left(\mu_{2}, \nu_{2}\right)$ and assume that there is a flux surface $\psi=\psi_{X}$ at which the corresponding gaps cross, $\omega_{*\left(\mu_{1}, \nu_{1}\right)}\left(\psi_{X}\right)=\omega_{*\left(\mu_{2}, \nu_{2}\right)}\left(\psi_{X}\right)$, which means due to equation (9) that $\tilde{k}_{* 1}\left(\psi_{X}\right)=\sigma \tilde{k}_{* 2}\left(\psi_{X}\right)$ with $\sigma= \pm 1$, where $\tilde{k}_{* 1} \equiv \tilde{k}_{*\left(\mu_{1}, \nu_{1}\right)}$ and $\tilde{k}_{* 2} \equiv \tilde{k}_{*\left(\mu_{2}, \nu_{2}\right)}$. This, in particular, implies that

$$
\iota_{X} \equiv \iota\left(\psi_{X}\right)=\frac{\nu_{1}-\sigma \nu_{2}}{\mu_{1}-\sigma \mu_{2}} N
$$

is rational, so that the field lines are closed at $\psi=\psi_{X}$.

Having in mind that equation (1) involves only derivatives along the field lines, we study this equation on a single closed field line, $\theta=\alpha+\iota\left(\psi_{X}\right) \phi$, where $\alpha=$ const is a field line label. Then, using equation (13), we put equation (4) into the form

$$
h_{g, c}^{\psi \psi}=1+\frac{1}{2} \epsilon_{g, c(X)}(\alpha) \exp \left(i \frac{\mu_{2} \nu_{1}-\mu_{1} \nu_{2}}{\mu_{1}-\sigma \mu_{2}} N \phi\right)+\text { c.c. }+(\ldots),
$$

where

$\epsilon_{g, c(X)}(\alpha)= \begin{cases}\epsilon_{g, c 1}^{\psi \psi} \exp \left(i \mu_{1} \alpha\right)+\epsilon_{g, c 2}^{\psi \psi} \exp \left(i \mu_{2} \alpha\right) & \text { when } \sigma=+1, \\ \left(\epsilon_{g, c 1}^{\psi \psi}\right)^{*} \exp \left(-i \mu_{1} \alpha\right)+\epsilon_{g, c 2}^{\psi \psi} \exp \left(i \mu_{2} \alpha\right) & \text { when } \sigma=-1,\end{cases}$ 
$\epsilon_{g, c 1}^{\psi \psi} \equiv \epsilon_{g, c\left(\mu_{1}, \nu_{1}\right)}^{\psi \psi}, \epsilon_{g, c 2}^{\psi \psi} \equiv \epsilon_{g, c\left(\mu_{2}, \nu_{2}\right)}^{\psi \psi}$, "c.c." means complex conjugate, and (...) stands for the contribution of all harmonics except for $(\mu, \nu)=\left( \pm \mu_{1}, \pm \nu_{1}\right)$ and $\left( \pm \mu_{2}, \pm \nu_{2}\right)$. We observe that the two harmonics merge into one on each field line. The amplitude of this net harmonic and, thus, the width of the resulting gap in the continuum depends on the field line (on $\alpha$ ). The total continuous spectrum is the union of the continua for all field lines. Therefore, neglecting the influence of the rest of harmonics and using equation (10), we can estimate the width of the joint gap produced by the harmonics $\left(\mu_{1}, \nu_{1}\right)$ and $\left(\mu_{2}, \nu_{2}\right)$ as

$$
\delta \omega \approx \frac{1}{2} \min _{\alpha}\left|\epsilon_{g(X)}(\alpha)+\epsilon_{c(X)}(\alpha)\right| \omega=|| \epsilon_{\left(\mu_{1}, \nu_{1}\right)}^{\psi \psi}|-| \epsilon_{\left(\mu_{2}, \nu_{2}\right)}^{\psi \psi}|| \omega .
$$

We conclude that the two gaps "annihilate" at the crossing point: the width of the net gap is approximately the difference of the widths that the two gaps would have if they were alone. Another conclusion we make is that at $\psi=\psi_{X}$ the continuum depends on the field line, the dependence being the stronger, the wider are the crossing gaps. This implies that the continuum wave functions are localized at separate field lines.

\subsection{Vicinity of the crossing: Numerical study}

To study the AC in the vicinity of a crossing point of two gaps numerically, we proceed from the matrix form of the AC equation (equation (6)). Let us choose a wave harmonic with the wave numbers $(m, n)=\left(m_{0}, n_{0}\right)$ such that $\tilde{k}_{m_{0}, n_{0}} \approx \tilde{k}_{* 1}$. Then we define a sequence of harmonics as follows:

$$
\begin{aligned}
& \left(m_{2 l+1}, n_{2 l+1}\right)=\left(m_{2 l}, n_{2 l}\right)-\left(\mu_{1}, \nu_{1} N\right), \\
& \left(m_{2 l+2}, n_{2 l+2}\right)=\left(m_{2 l+1}, n_{2 l+1}\right)+\sigma\left(\mu_{2}, \nu_{2} N\right) .
\end{aligned}
$$

Since $\tilde{k}_{* 1} \approx \sigma \tilde{k}_{* 2}$ near the crossing point of the gaps, a large number of the harmonics of the sequence are characterized there by approximately the same longitudinal wave numbers (up to sign):

$$
\ldots \approx-\tilde{k}_{m_{-1}, n_{-1}} \approx \tilde{k}_{m_{0}, n_{0}} \approx-\tilde{k}_{m_{1}, n_{1}} \approx \tilde{k}_{m_{2}, n_{2}} \approx \ldots
$$

Therefore, one can expect the interaction of the harmonics within the sequence to be strong, whereas the coupling with harmonics outside the sequence can be expected to be much weaker. Having this in mind, we simplify equation (6) by taking the sub-matrix corresponding to harmonics $\left(m_{s}, n_{s}\right)$ with $s=-L, \ldots, L$. In addition, we neglect all coupling parameters except for $(\mu, \nu)=\left(\mu_{1}, \nu_{1}\right),\left(\mu_{2}, \nu_{2}\right),\left(-\mu_{1},-\nu_{1}\right)$, and $\left(-\mu_{2},-\nu_{2}\right)$. We obtain the following eigenvalue problem for three-diagonal matrices:

$$
\sum_{s=-L}^{L} \mathcal{G}_{p, s}(d, \kappa) \Phi_{s}=\Omega^{2} \sum_{s=-L}^{L} \mathcal{C}_{p, s} \Phi_{s}, \quad s=-L, \ldots, L
$$


where $\Phi_{s}$ is the wave harmonic with the wave numbers $\left(m_{s}, n_{s}\right)$, the three-diagonal matrices $\mathcal{G}$ and $\mathcal{C}$ are given by

$$
\mathcal{G}_{p, s}=\mathcal{G}_{s, p}^{*}=\tilde{k}_{p} \tilde{k}_{s} \begin{cases}1 & \text { for } p=s, \\ \epsilon_{g 1}^{\psi \psi} & \text { for } p=s-1=2 l-1, \\ \epsilon_{g\left(-\sigma \mu_{2},-\sigma \nu_{2}\right)}^{\psi \psi} & \text { for } p=s-1=2 l, \\ 0 & \text { for } p \neq s, s \pm 1,\end{cases}
$$

$$
\mathcal{C}_{p, s}=\mathcal{C}_{s, p}^{*}= \begin{cases}1 & \text { for } p=s, \\ \epsilon_{c 1}^{\psi \psi} & \text { for } p=s-1=2 l-1, \\ \epsilon_{c\left(-\sigma \mu_{2},-\sigma \nu_{2}\right)}^{\psi \psi} & \text { for } p=s-1=2 l, \\ 0 & \text { for } p \neq s, s \pm 1,\end{cases}
$$

$\tilde{k}_{p} \equiv \tilde{k}_{m_{p}, n_{p}}=(-1)^{p} \tilde{k}_{X}+\kappa+p d, d(\psi)=\sigma \tilde{k}_{* 2}-\tilde{k}_{* 1}, \tilde{k}_{X}(\psi)=\left(\sigma \tilde{k}_{* 2}+\tilde{k}_{* 1}\right) / 2$, $\kappa=\tilde{k}_{m_{0}, n_{0}}-\tilde{k}_{X}$ is a parameter.

For the exact crossing $\left(\iota=\iota_{X}, d=0\right)$, equation (19) for $L=\infty$ can be solved analytically. Indeed, the matrices are periodic in this case: $\mathcal{G}_{p+2, s+2}=\mathcal{G}_{p, s}$, $\mathcal{C}_{p+2, s+2}=\mathcal{C}_{p, s}$. Then we can take $\Phi_{s}$ in a periodic form:

$$
\Phi_{2 l}=a_{1} \exp (i \gamma l), \quad \Phi_{2 l+1}=a_{2} \exp (i \gamma l) .
$$

Solving the obtained eigenvalue problem and varying $\kappa$ and $\gamma$, we obtain a continuous spectrum with a gap, the boundaries of the gap corresponding to $\kappa=0$. The width of the gap agrees with equation (16). Thus, as one could expect, we recover results of subsection 2.2 (the gap annihilation). Note that the eigenfunction described by equation (22) is localized on a field line (which depends on $\gamma$ ), also in agreement with subsection 2.2 .

To study the spectrum for $\iota \neq \iota_{X}$, we solved the eigenvalue problem (19) numerically with the use of the QZ algorithm [33]. In each calculation we obtained several eigenvectors peaked at $l \approx \pm L$. Such eigenvectors were discarded as spurious. The rest of the eigenvectors seemed to converge with the increase of $L$, although with different rates. To outline the continuum for some $\iota$, we scanned $\kappa \equiv \tilde{k}_{m_{0}, n_{0}}$ with a small step in the interval $-\delta \leq \kappa \leq \delta$ (it is sufficient to take $\kappa$ within this interval because $\tilde{k}_{2 l}-\tilde{k}_{X}$ for some $l$ always lies in this interval).

Two typical results of the calculations are presented in figures 3 and 4, where the spectra of equation (19) are shown versus $d$ (note that $d$ can be considered as a radial variable when $\iota$ is a monotonic function of $\psi$ ). The eigenvalues of equation (19) are shown with dots, which form vertical bands as $\kappa$ is varied. We observe that the gaps indeed annihilate at the crossing point $(d=0)$. In particular, in figure 3 , where a crossing of two gap of equal widths is shown, the annihilation is total (both gaps are closed at $d=0)$. In the vicinity of the crossing point, multiple combination gaps appear, which are characterized by the coupling numbers $2\left(\mu_{1}, \nu_{1}\right)-\sigma\left(\mu_{2}, \nu_{2}\right), 3\left(\mu_{1}, \nu_{1}\right)-2 \sigma\left(\mu_{2}, \nu_{2}\right)$, etc. The widths of such gaps decrease with the increase of $d$, so that at the ends of the 


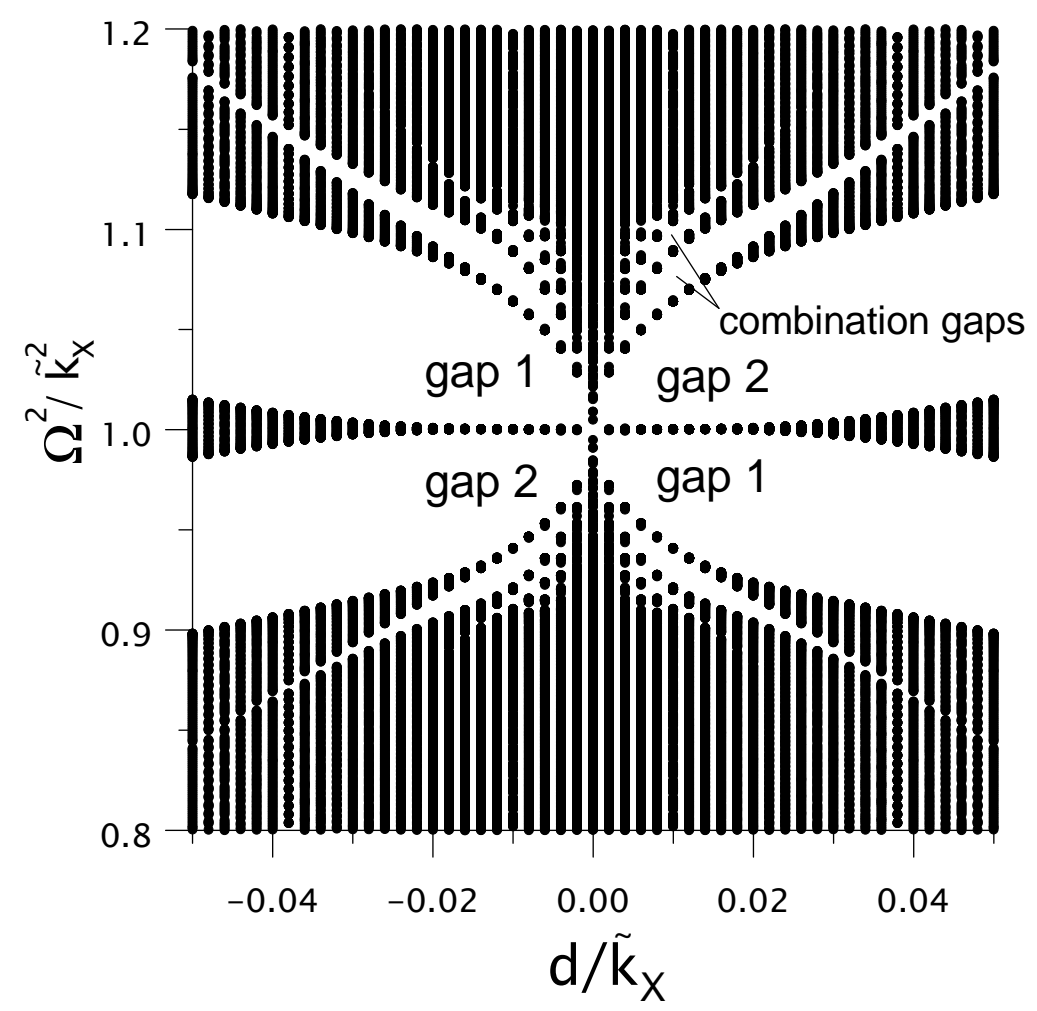

Figure 3. Alfvén continuum near a crossing of two gaps of equal width: $\epsilon_{g 1}^{\psi \psi}=\epsilon_{g 2}^{\psi \psi}=$ $0, \epsilon_{c 1}^{\psi \psi}=\epsilon_{c 2}^{\psi \psi}=0.05, L=20$.

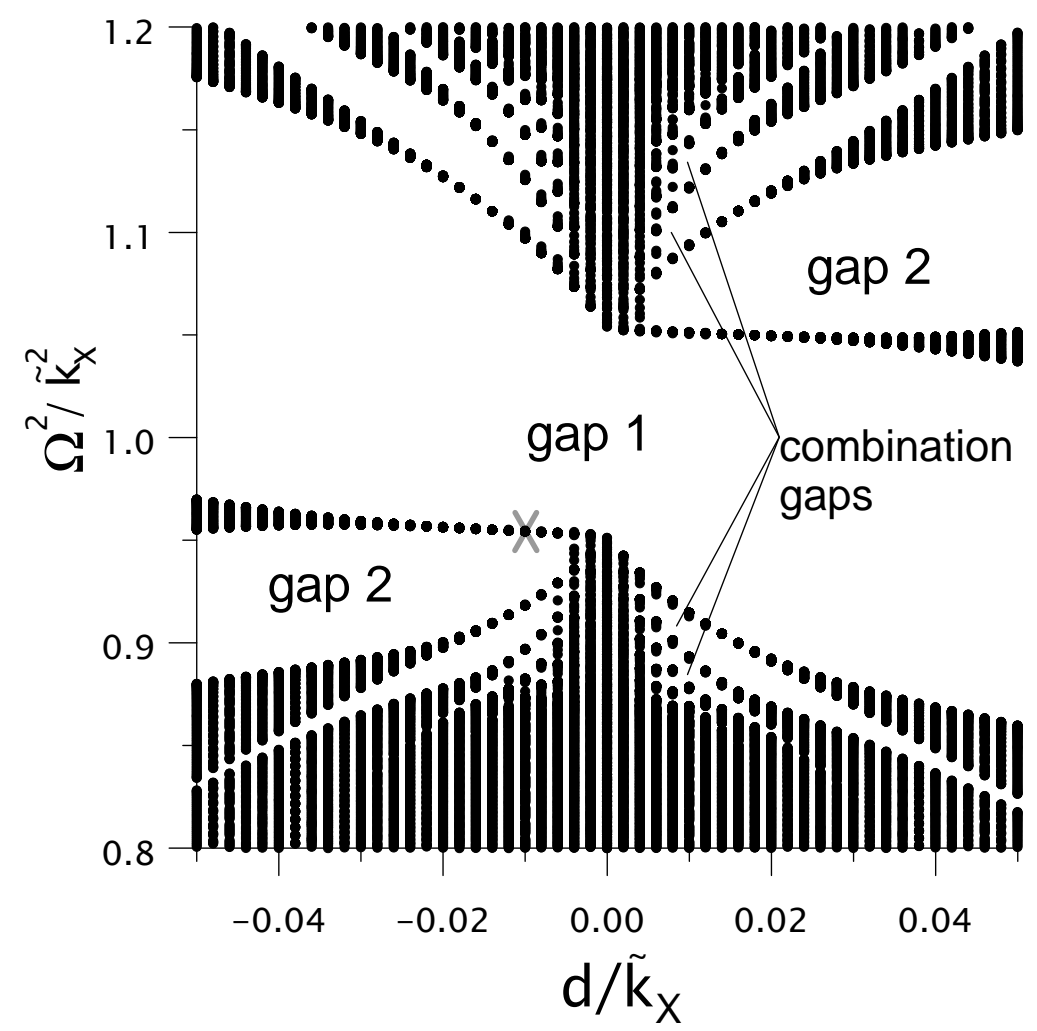

Figure 4. Alfvén continuum near a crossing of two gaps, one of them being twice wider than the other: $\epsilon_{g 1}^{\psi \psi}=\epsilon_{g 2}^{\psi \psi}=0, \epsilon_{c 1}^{\psi \psi}=0.1, \epsilon_{c 2}^{\psi \psi}=0.05, L=20$. 
interval most of them are negligible. The gaps are separated by continuum walls, which are extremely thin at small $d$, e.g., $\delta \Omega / \Omega \approx 3 \times 10^{-8}$ for the continuum wall between the main gaps at $d=-0.005$ in figure 4 (at the place shown with gray cross). The width of each wall decreases with a decrease of $d$, obeying the law $\propto \exp (-C / d)$, where $C$ is a constant depending on the specific wall. Calculations with increased $L$ reveal more combination gaps because the approximation error because of finite $L$ increases the width of the walls.

Note that the Fourier structure of the continuum wave functions becomes very complicated as $d$ decreases (the eigenfunctions at the exact crossing consist of infinite number of harmonics). This explains the computational difficulties observed in continuum calculations [34] near the flux surfaces where pairs of wide gaps cross.

It should be mentioned also that the characteristic picture shown in figures 3 and 4 is not a result of the approximations we made (only two harmonics in the equilibrium and a sequence of harmonics described by equation (17) are taken into account). Similar picture was obtained in [34] (figure 5), where no such assumptions were made. These assumptions were needed here only to reach better numerical resolution.

\subsection{Vicinity of the crossing: Analytical solution}

We solve equation (2) in a vicinity of a gap crossing, assuming that the coefficients include only cosine harmonics (this is typically the case in stellarators) and keeping only the harmonics $\left(\mu_{1}, \nu_{1}\right)$ and $\left(\mu_{2}, \nu_{2}\right)$ responsible for the crossing gaps. Expressing the coupling numbers in terms of $\tilde{k}_{X}$ and $d$, we obtain:

$h_{g, c}^{\psi \psi}=1+\epsilon_{g, c 1}^{\psi \psi} \cos \left[\left(2 \tilde{k}_{X}-d\right) \phi+\mu_{1} \alpha\right]+\epsilon_{g, c 2}^{\psi \psi} \cos \left[\sigma\left(2 \tilde{k}_{X}+d\right) \phi+\mu_{2} \alpha\right]$,

where the coupling parameters are assumed to be real. When $v \equiv d / \tilde{k}_{X} \ll 1$, the periods of both harmonics almost coincide. Then the functions $h_{g}^{\psi \psi}(\phi)$ and $h_{c}^{\psi \psi}(\phi)$ have two characteristic scales. The fast scale, $\Delta \phi \sim \pi / \tilde{k}_{X}$, is approximately the period of both harmonics. The slow one, $\Delta \phi \sim \pi / d$, is the period of the slow variation of the relative phase of the two harmonics.

Before a rigorous analysis, we will give a graphical explanation of the results that we are going to obtain. In figure 5, a sketch of the variation of $h=\left(h_{g}^{\psi \psi}+h_{c}^{\psi \psi}\right) / 2$ along a field line is presented. When $v \ll 1$, the sum of the two harmonics entering $h$ can be considered as one harmonic with the amplitude slowly varying between $\epsilon_{\max }=$ $\left|\epsilon_{1}^{\psi \psi}\right|+\left|\epsilon_{2}^{\psi \psi}\right|$ and $\epsilon_{\min }=|| \epsilon_{1}^{\psi \psi}|-| \epsilon_{2}^{\psi \psi}||$, where $\epsilon_{i}^{\psi \psi}=\left(\epsilon_{g i}^{\psi \psi}+\epsilon_{c i}^{\psi \psi}\right) / 2$ with $i=1,2$. As follows from equation (10), the amplitude of the oscillations of a certain harmonic of $h$ along the field lines characterizes the width of the corresponding continuum gap. Assuming that the variation of the amplitude is sufficiently slow to be neglected, we conclude that the continuum wave propagation is forbidden for $1-\epsilon_{\min }<\Omega^{2} / \tilde{k}_{X}^{2}<1+\epsilon_{\min }$, whereas the frequencies outside the interval $1-\epsilon_{\max }<\Omega^{2} / \tilde{k}_{X}^{2}<1+\epsilon_{\max }$ belong to the continuum. In the intermediate intervals, $1-\epsilon_{\max }<\Omega^{2} / \tilde{k}_{X}^{2}<1-\epsilon_{\min }$ and $1+\epsilon_{\min }<\Omega^{2} / \tilde{k}_{X}^{2}<1+\epsilon_{\max }$, the wave is trapped in certain parts of the field lines, the parts where the frequency lies 


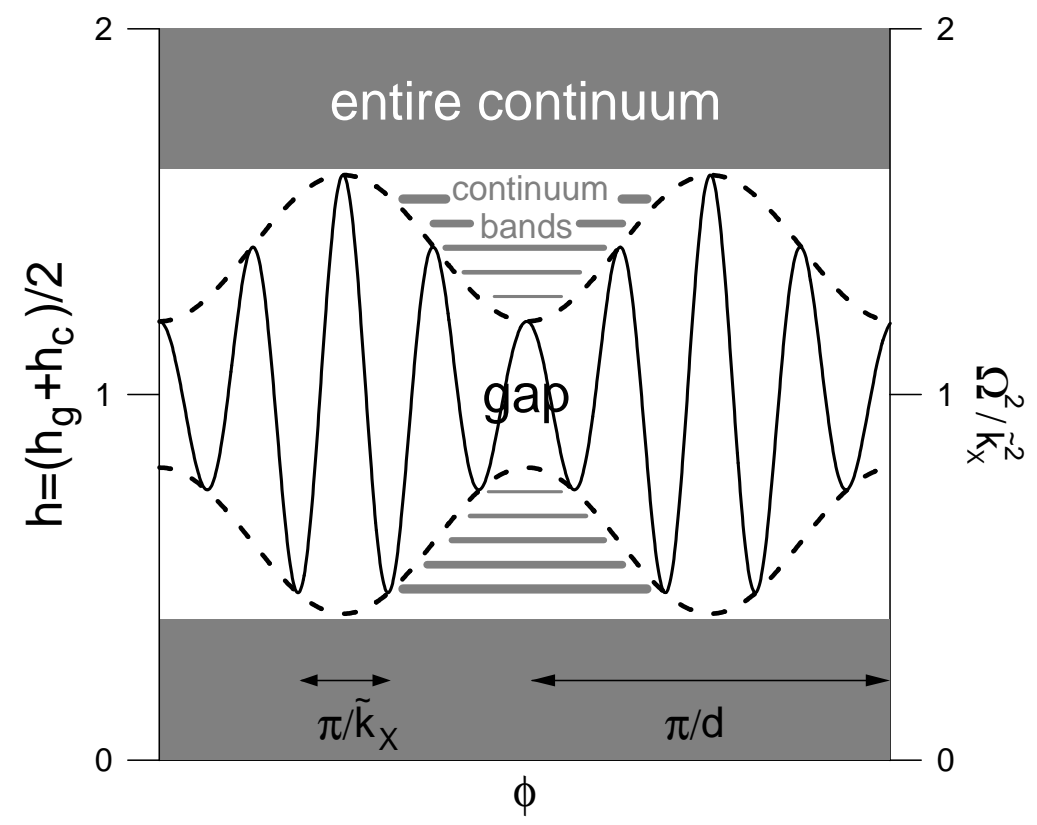

Figure 5. Sketch of the AC produced by two harmonics of the configuration with close characteristic longitudinal wave numbers. The variation $h=\left(h_{g}^{\psi \psi}+h_{c}^{\psi \psi}\right) / 2$ along a field line is also shown. Solid curve, $h$; dashed curves, the envelopes of $h$; grey horizontal bands, the continuum. The region between the envelopes is a forbidden zone for the continuum waves.

outside the envelopes of $h$ (see figure 5). This should result in the formation of a system of frequency levels. Similar levels in different "pockets" of the envelopes interact due to tunnelling, turning into continuum bands of finite width. We can assume that these bands correspond to the thin continuum walls observed in numerical calculations in subsection 2.3 (see figures 3 and 4). In a sense, such continua are similar to deep electron energy zones in a solid crystal. The energy of such a zone can be found in the assumption that the electron is bounded in a single potential well corresponding to a single atom. However, tunneling between the atoms results in the zone width being finite.

Now we proceed to quantitative calculations. We begin with averaging equation (2) over the fast scale. With this aim, we present it in a Hamiltonian form:

$$
\frac{\mathrm{d} \Phi}{\mathrm{d} t}=\frac{\partial H}{\partial P}, \quad \frac{\mathrm{d} P}{\mathrm{~d} t}=-\frac{\partial H}{\partial \Phi},
$$

where

$$
\begin{gathered}
H=\frac{1}{2 h_{g}^{\psi \psi}} P^{2}+\frac{1+\lambda}{2} h_{c}^{\psi \psi} \Phi^{2} \approx \frac{1}{2}\left(P^{2}+\Phi^{2}\right)+\frac{1}{2}\left[-\chi_{g} P^{2}+\left(\lambda+\chi_{c}\right) \Phi^{2}\right]+\mathrm{O}\left(\epsilon^{2}\right), \\
P=\frac{h_{g}^{\psi \psi}}{\tilde{k}_{X}} \frac{\mathrm{d} \Phi}{\mathrm{d} \phi}
\end{gathered}
$$

$t=\tilde{k}_{X} \phi, \lambda=\Omega^{2} / \tilde{k}_{X}^{2}-1, \chi_{g, c}=h_{g, c}^{\psi \psi}-1$. We assume that $v \ll 1$ and $\lambda \sim \chi_{g} \sim \chi_{c} \sim \epsilon$, where $\epsilon$ is a small parameter. Our aim is to average the fast oscillations in the first-order 
part of the Hamiltonian along the trajectories of the zeroth-order motion. One can see that these trajectories rotate with the unit frequency around the origin of the phase plane $(\Phi, P)$. Having this in mind, we introduce the action-angle coordinates $(\zeta, J)$ as follows:

$$
\Phi=(2 J)^{1 / 2} \sin (\zeta+t), \quad P=(2 J)^{1 / 2} \cos (\zeta+t) .
$$

The coordinate transformation $(\Phi, P) \rightarrow(\zeta, J)$ is canonical, resulting from the generating function $\Phi^{2} \cot (\zeta+t) / 2$. To first order, the Hamiltonian in the new coordinates is

$$
\hat{H}=H-J=\left[-\chi_{g} \cos ^{2}(\zeta+t)+\left(\lambda+\chi_{c}\right) \sin ^{2}(\zeta+t)\right] J
$$

The zero-order trajectories in the new coordinates are simply $J=$ const, $\zeta=$ const. Therefore, the averaging consists in putting $h_{g}$ and $h_{c}$ given by equation (23) to equation (28) and excluding fast oscillations with $\mathrm{d} / \mathrm{d} t \sim 1$. This can be considered as a transformation from the variables $(\zeta, J)$ to new "corrected" canonical variables $(\bar{\zeta}, \bar{J})$, in which the Hamiltonian is free of fast oscillations [35] (detailed analysis shows that $\bar{J}=J+\mathcal{O}(\epsilon v), \bar{\zeta}=\zeta+\mathcal{O}(\epsilon v))$. We arrive at the Hamiltonian

$\bar{H}=\frac{1}{2}\left\{\lambda+g_{-} \cos [2(\bar{\zeta}+z)] \cos (v \hat{t})+g_{+} \cos [2(\bar{\zeta}+z)] \cos (v \hat{t})\right\} \bar{J}$,

where $\hat{t}=t-t_{M}$, the time shift $t_{M}=\left[\left(\mu_{1}-\sigma \mu_{2}\right) \alpha+\arccos (-s)+2 \pi M\right] /(2 v)$ is defined so that any local minimum of the amplitude of $h$ could be placed to $\hat{t}=0$ with an appropriate choice of integer $M, z=\left(v t_{0}-\mu_{1} \alpha\right) / 2, g_{-}=(-1)^{M}\left(s \epsilon_{2}^{\psi \psi}-\epsilon_{1}^{\psi \psi}\right)$, $g_{+}=(-1)^{M}\left(s \epsilon_{2}^{\psi \psi}+\epsilon_{1}^{\psi \psi}\right), s=\operatorname{sgn}\left(\epsilon_{1}^{\psi \psi} \epsilon_{2}^{\psi \psi}\right)$. Introducing new coordinates, $(\bar{\Phi}, \bar{P})$, with the canonical transformation

$$
\bar{\Phi}=(2 \bar{J})^{1 / 2} \sin (\bar{\zeta}+z), \quad \bar{P}=(2 \bar{J})^{1 / 2} \cos (\bar{\zeta}+z),
$$

we obtain

$$
\bar{H}=\frac{1}{4}\left[A(\hat{t}) \bar{\Phi}^{2}+B(\hat{t}) \bar{P}^{2}+2 g_{+} \sin (v \hat{t}) \bar{P} \bar{\Phi}\right],
$$

where $A=\lambda-g_{-} \cos (v \hat{t}), B=\lambda+g_{-} \cos (v \hat{t})$.

The Hamiltonian of the form (31) can be reduced to Schrödinger equations. To achieve this, we perform the canonical transformations

$$
\begin{array}{ll}
P_{1}=|B|^{1 / 2} \bar{P}, & \Phi_{1}=|B|^{-1 / 2} \bar{\Phi}, \\
P_{2}=|A|^{-1 / 2} \bar{P}, & \Phi_{2}=|A|^{1 / 2} \bar{\Phi}
\end{array}
$$

to obtain the following pair of Hamiltonians:

$$
\begin{aligned}
& H_{1}=\frac{1}{4}\left\{\varsigma_{1} A B \Phi_{1}^{2}+\varsigma_{1} P_{1}^{2}+\left[2 g_{+} \sin (v \hat{t})-\frac{2}{B} \frac{\mathrm{d} B}{\mathrm{~d} \hat{t}}\right] P_{1} \Phi_{1}\right\}, \\
& H_{2}=\frac{1}{4}\left\{\varsigma_{2} \Phi_{2}^{2}+\varsigma_{2} A B P_{2}^{2}+\left[2 g_{+} \sin (v \hat{t})+\frac{2}{A} \frac{\mathrm{d} A}{\mathrm{~d} \hat{t}}\right] P_{2} \Phi_{2}\right\},
\end{aligned}
$$


where $\varsigma_{1}=\operatorname{sgn}(B), \varsigma_{2}=\operatorname{sgn}(A)$. Excluding one of the variables from the resulting Hamilton's equations, we obtain a pair of Schrödinger equations. Omitting the terms containing $v^{2}$ or $v \sin (v \hat{t})$, we can write these equations as follows:

$$
\begin{aligned}
& \frac{\mathrm{d}^{2} \Phi_{1}}{\mathrm{~d} \hat{t}^{2}}+\left[\frac{\lambda^{2}-g_{-}^{2}}{4}-\frac{g_{+}^{2}-g_{-}^{2}}{4} \sin ^{2}(v \hat{t})-\frac{g_{+} v}{2} \cos (v \hat{t})\right] \Phi_{1}=0, \\
& \frac{\mathrm{d}^{2} P_{2}}{\mathrm{~d} \hat{t}^{2}}+\left[\frac{\lambda^{2}-g_{-}^{2}}{4}-\frac{g_{+}^{2}-g_{-}^{2}}{4} \sin ^{2}(v \hat{t})+\frac{g_{+} v}{2} \cos (v \hat{t})\right] P_{2}=0 .
\end{aligned}
$$

These two equations are complementary: We cannot use the first equation when $A \approx 0$ (i.e., $\lambda \approx g_{-} \cos (v \hat{t})$ ) and the second one when $B \approx 0$ (i.e., $\lambda \approx-g_{-} \cos (v \hat{t})$ ) because then some omitted terms of the equations are not small.

We observe that the averaging has confirmed the conclusions at which we arrived in our qualitative analysis. Indeed, we have obtained Schrödinger equations with periodic potentials, the wells of the potentials being at the same places as the "pockets" of the envelope of $h$. When the wells are deep enough, the spectrum of such a potential is, in fact, the discrete spectrum of a single well with the levels turning into narrow continuum bands due to tunnelling between the wells. Therefore, we solve equations (34) for a single potential well, disregarding the interaction between different wells. It is sufficient to consider a vicinity of $\hat{t}=0$ since we can place any well to that point by a proper choice of $M$. We assume that $|v \hat{t}| \sim\left|v / g_{+}\right|^{1 / 2} \ll 1$ (this estimate will be justified a posteriori), expand sine and cosine and omit terms of order $v^{2}$. We arrive at the following pair of quantum oscillator equations:

$$
\begin{array}{ll}
\frac{\mathrm{d}^{2} \Phi_{1}}{\mathrm{~d} \hat{t}^{2}}+\left(\frac{\lambda^{2}-g_{-}^{2}}{4}-\frac{g_{+} v}{2}-\frac{g_{+}^{2}-g_{-}^{2}}{4} v^{2} \hat{t}^{2}\right) \Phi_{1}=0, & \operatorname{sgn}(\lambda)=\operatorname{sgn}\left(g_{-}\right), \\
\frac{\mathrm{d}^{2} P_{2}}{\mathrm{~d} \hat{t}^{2}}+\left(\frac{\lambda^{2}-g_{-}^{2}}{4}+\frac{g_{+} v}{2}-\frac{g_{+}^{2}-g_{-}^{2}}{4} v^{2} \hat{t}^{2}\right) P_{2}=0, & \operatorname{sgn}(\lambda)=-\operatorname{sgn}\left(g_{-}\right) .
\end{array}
$$

The joint spectrum of these two equations is given by

$$
\frac{1}{2}\left(\lambda^{2}-g_{-}^{2}\right)+\operatorname{sgn}\left(\lambda g_{-}\right) g_{+} v=|v| \gamma(2 j+1), \quad j=0,1, \ldots
$$

with $\gamma=\left(g_{+}^{2}-g_{-}^{2}\right)^{1 / 2}$. Solving equation (36) for $\lambda$, we can write the frequencies of the continuum bands as follows:

$$
\begin{aligned}
& \lambda_{0}=-\operatorname{sgn}\left(g_{+} v\right) g_{-}\left[1-\frac{2|v|}{\gamma+\left|g_{+}\right|}\right]^{1 / 2}, \\
& \lambda_{ \pm j}= \pm\left[g_{-}^{2}+4 j \gamma|v| \pm \operatorname{sgn}\left(g_{+} g_{-} v\right) \frac{2|v| g_{-}^{2}}{\gamma+\left|g_{+}\right|}\right]^{1 / 2}, \quad j=1,2, \ldots
\end{aligned}
$$

(note that $j$ in this equation may differ by 1 from $j$ in equation (36)). One can see that the structure of the bands is in agreement with figures 3 and 4 . Note that the eigenfunctions in neighboring wells are different (because the substitution $M \rightarrow M+1$ results in $g_{+} \rightarrow-g_{+}$and $g_{-} \rightarrow-g_{-}$), but the eigenfrequencies are the same.

The obtained solutions are valid when the tunnelling between different potential wells is weak enough, i.e., when the distance between the turning points is much less 
than the potential period, which is $2 \pi /|v|$. Putting the frequency levels determined by equation (36) into equation (35) and taking in to account that $g_{+}^{2}-g_{-}^{2}=4\left|\epsilon_{1}^{\psi \psi} \epsilon_{2}^{\psi \psi}\right|$, we observe that the region of the wave propagation is

$$
|\hat{t}| \leq \Delta t=\frac{C}{\left|\epsilon_{1}^{\psi \psi} \epsilon_{2}^{\psi \psi}\right|^{1 / 4}|v|^{1 / 2}}
$$

with $C=(2 j+1)^{1 / 2} \sim 1$. Thus, the tunneling is weak when

$$
|v|=2 \frac{|| \tilde{k}_{* 1}|-| \tilde{k}_{* 2}||}{\left|\tilde{k}_{* 1}\right|+\left|\tilde{k}_{* 2}\right|}=2 \frac{\left|\Omega_{* 1}-\Omega_{* 2}\right|}{\Omega_{* 1}+\Omega_{* 2}} \ll \frac{\pi^{2}}{4}\left|\epsilon_{1}^{\psi \psi} \epsilon_{2}^{\psi \psi}\right|^{1 / 2} .
$$

Using the similarity with electron waves in a crystal, we conclude that in this case the width of a continuum band depends on $v$ as $\sim \exp (-D /|v|)$ with $D$ constant, where the argument of the exponent originates from the width of a potential barrier between the wells. This dependence is also in agreement with the numerical results of subsection 2.3.

Let us formulate our results in physical terms. The inequality (39) means that the relative difference between the periods of the harmonics along the field lines is small in comparison with the corresponding coupling parameters. At the same time, this inequality requires that the estimated distance between the gaps should be smaller than their estimated widths, which is exactly the situation that we intended to analyze in subsection 2.1. When it is satisfied for a certain pair of equilibrium Fourier harmonics, the continuum wave functions are localized in the regions given by equation (38). Returning from $\bar{t}$ to the angles $\theta$ and $\phi$ in equation (38), we observe that the wave is trapped within certain "waveguides" on each flux surface:

$$
\left|\left(\sigma \mu_{2}-\mu_{1}\right) \theta-\left(\sigma \nu_{2}-\nu_{1}\right) \phi-\arccos (-s)+2 \pi M\right| \leq \frac{2 C|v|^{1 / 2}}{\left|\epsilon_{1}^{\psi \psi} \epsilon_{2}^{\psi \psi}\right|^{1 / 4}} .
$$

These waveguides are produced by the interference of the equilibrium harmonics with close periods along the magnetic field and are located at the places where the harmonics tend to cancel. Equation (40) shows that the width of the waveguides decreases when we approach the gap crossing $(v \rightarrow 0)$. The continuum in this case looks as a set of narrow frequency bands. The width of such a band is determined by the tunnelling through the evanescence zones between the waveguides and exponentially decreases with $|v|^{-1}$.

The analysis above sheds light on the mentioned peculiarities of the structure of the high-frequency part of the AC in stellarators. As mentioned in subsection 2.1, the dominant Fourier harmonics of the magnetic configuration in many stellarators (including W7-AS) are $(\mu, \nu)=(2,1)$ and $(3,1)$, which are associated with helical elongation and triangularity of the plasma cross section. The other helicity- and mirrorinduced gaps can be considered, at least, partly as combination gaps created by these harmonics $((4,1)=2 \times(3,1)-(2,1) ;(0,1)=3 \times(2,1)-2 \times(3,1)$, etc. $)$. Indeed, the width of some the helicity-induced gaps is too large to be explained by the corresponding coupling parameters. It follows from equations (11) and (12) that the condition (39) is satisfied; hence, the continuum wave functions in the vicinity of these gaps are trapped. Taking into account that $\operatorname{sgn}\left(\epsilon_{(2,1)}^{\psi \psi}\right)=\operatorname{sgn}\left(\epsilon_{(3,1)}^{\psi \psi}\right)$ (which seems typical in other stellarators, too), we find from equation (40) that the waves are trapped at the inner 
circumference of the torus, near $\theta=\pi$, which agrees with the observed anti-ballooning of the wave functions (figure 2). The extremely small widths of the continuum walls between the gaps in this part of the spectrum (see subsection 2.1) are explained by their exponential dependence on $|v|^{-1}$ (the latter is about 8 for the gaps $(2,1)$ and $(3,1)$, see equation (12)).

\section{Alfvén eigenmodes produced by interaction of two close gaps}

It is well known that the dependence of an $\mathrm{AE}$ on the poloidal and toroidal angles is usually similar to that of the continuum branches with which the AE is associated (e.g., this is so for the odd and even TAE modes). Therefore, it is of interest to check if the trapping of continuum waves in waveguides takes places for AEs in the same frequency range. Neglecting the plasma pressure, we proceed from the following equation of ideal Alfvén oscillations [36, 19]:

$\sum_{i, j}\left\{\frac{1}{g B^{2}} \hat{L} \frac{\partial}{\partial x^{i}}\left[\sqrt{g} g_{\perp}^{i j} B^{2} \frac{\partial}{\partial x^{j}}\left(\frac{\hat{L} \Phi}{B^{2} \sqrt{g}}\right)\right]+\frac{1}{\sqrt{g}} \frac{\partial}{\partial x^{i}}\left(\sqrt{g} g_{\perp}^{i j} \frac{\omega^{2}}{v_{A}^{2}} \frac{\partial \Phi}{\partial x^{i}}\right)\right\}=0$,

where $g_{\perp}^{i j}=g^{i j}-b^{i} b^{j} ; g^{i j}$ with $i, j=\psi, \theta, \phi$ are the components of the contravariant metric tensor; $b^{i}$ denotes the corresponding contravariant component of $\boldsymbol{b}=\boldsymbol{B} / B$. In a large-aspect-ratio device, the components $g^{i \phi}=g^{\phi i}$ with $i=\psi, \theta, \phi$ are small (of order of the squared inverse aspect ratio) in comparison with the rest [19]; therefore, below we neglect the terms with $i=\phi$ or $j=\phi$ in equation (41).

To treat equation (41), we use the ballooning formalism as described in [26]. We take $\Phi$ in the eikonal form,

$$
\Phi=\hat{\Phi}(\psi, \theta, \phi) \exp \left[i \varepsilon^{-1} S(\psi, \alpha)\right],
$$

where $\alpha=\theta-\iota \phi, \varepsilon$ is a small parameter indicating that we consider waves with $k_{\|} \ll k_{\perp}$, the eikonal $S(\psi, \alpha)$ is constant along the field lines, and the function $\hat{\Phi}$ characterizes both the variation of the wave along the field lines and the slow transversal variation of the wave envelope.

Putting the ballooning ansatz (42) into equation (41) and collecting the terms of lowest order in $\varepsilon$, we obtain the ballooning equation:

$$
\frac{\mathrm{d}}{\mathrm{d} \phi}\left[\Delta\left(\phi ; \psi, \alpha, \phi_{k}\right) \frac{\mathrm{d} \hat{\Phi}}{\mathrm{d} \phi}\right]+\Omega^{2} \frac{\Delta\left(\phi ; \psi, \alpha, \phi_{k}\right)}{h_{B}^{4}(\phi ; \psi, \alpha)} \hat{\Phi}=0,
$$

where

$$
\Delta=h_{g}^{\theta \theta}+2\left(\phi-\phi_{k}\right) \hat{s} \iota h_{g}^{\psi \theta}+\left(\phi-\phi_{k}\right)^{2} \hat{s}^{2} \iota^{2} h_{g}^{\psi \psi}
$$

$h_{g}^{\theta \theta}=g_{\perp}^{\theta \theta} /\left\langle g_{\perp}^{\theta \theta}\right\rangle$ and $h_{g}^{\psi \theta}=g^{\psi \theta} /\left(\left\langle g^{\psi \psi}\right\rangle\left\langle g_{\perp}^{\theta \theta}\right\rangle\right)^{1 / 2}$ are normalized metric tensor components, $\hat{s}=-\iota^{\prime}\left(\left\langle g^{\psi \psi}\right\rangle /\left\langle g^{\theta \theta}\right\rangle\right)^{1 / 2} / \iota$ is the magnetic shear, prime means differentiation in $\psi$, $\phi_{k}=k_{\iota} / k_{\alpha}, k_{\iota}$ and $k_{\alpha}$ are transversal wave numbers introduced by the equation $\nabla S=k_{\alpha} \nabla \alpha+k_{\iota} \nabla \iota$. The ballooning equation is an ordinary differential equation, which determines the behaviour of the wave function along a field line. The coefficients 
of this equation are taken along the field line, so that $\theta=\theta(\psi, \alpha, \phi)=\alpha+\iota(\psi) \phi$ with $\psi, \alpha=$ const. The quantity $\phi_{k}$ plays the part of a parameter in this equation, as well as $\psi$ and $\alpha$. The dependence of the solution on these quantities is established through high-order analysis $[26,37,38]$. Such analysis enables one, in particular, to obtain the socalled "quantization condition," which provides the periodicity of the obtained solution in $\theta$ and $\phi$. One can see that the ballooning equation is reduced to the continuum equation (2) in the limit of $\phi \rightarrow \pm \infty$. Below we omit hats over $\Phi$ since this will not lead to a confusion.

Like in section 2, we assume for the sake of simplicity that the metric coefficients of the magnetic configuration include only two Fourier harmonics, $\left(\mu_{1}, \nu_{1}\right)$ and $\left(\mu_{2}, \nu_{2}\right)$. Then we present $h_{g}^{\psi \theta}$ and $h_{g}^{\theta \theta}$ as follows (cf. equation (23)):

$$
\begin{aligned}
& h_{g}^{\theta \theta}=1+\epsilon_{g 1}^{\theta \theta} \cos \left[\tilde{k}_{X}(2-v) \phi+\mu_{1} \alpha\right]+\epsilon_{g 2}^{\theta \theta} \cos \left[\tilde{k}_{X} \sigma(2+v) \phi+\mu_{2} \alpha\right], \\
& h_{g}^{\psi \theta}=\epsilon_{g 1}^{\psi \theta} \sin \left[\tilde{k}_{X}(2-v) \phi+\mu_{1} \alpha\right]+\epsilon_{g 2}^{\psi \theta} \sin \left[\sigma\left(2 \tilde{k}_{X}+d\right) \phi+\mu_{2} \alpha\right],
\end{aligned}
$$

where $v \ll 1$ (we take into account that $\left\langle g^{\psi \theta}\right\rangle=0$ ). On substituting equations (23), (45) and (46) to equation (44), we find that the coefficients of equation (43) possess three characteristic scales. Two of them, $\Delta \phi \sim \pi / \tilde{k}_{X}$ (the period of the harmonics) and $\Delta \phi \sim \pi /\left(v \tilde{k}_{X}\right)$ (the period of the beatings), have already appeared in our treatment of the continuum equation. The third one, $\Delta \phi \sim 2 /(\iota \hat{s})$, is a characteristic scale that usually appears in the ballooning theory. We consider the case of $v \ll 1$ and $\bar{s}=\hat{s} \iota / \tilde{k}_{X} \ll 1$, when the first scale is much shorter than the other two and use the procedure described in subsection 2.4 to average equation (43) over fast oscillations.

Equation (43) is equivalent to Hamilton's equations resulting from the Hamiltonian

$$
H(P, \Phi, t)=\frac{1}{2 \Delta} P^{2}+(1+\lambda) \frac{\Delta}{2 h_{B}^{4}} \Phi^{2}
$$

where $P=\Delta \mathrm{d} \Phi / \mathrm{d} t, \lambda=\Omega^{2} / \tilde{k}_{X}^{2}-1$ will be assumed to be $\sim \epsilon$, and the time variable is $t=\tilde{k}_{X} \phi$. Transforming this Hamiltonian to action-angle variables $(\zeta, J)$ with the canonical transformation $\Phi=(2 J / \Delta)^{1 / 2} \sin (\zeta+t), P=(2 J \Delta)^{1 / 2} \cos (\zeta+t)$, we obtain the Hamiltonian

$$
H(J, \zeta, t)=\frac{\lambda+1-h_{B}^{4}}{h_{B}^{4}} J \sin ^{2}(\zeta+t)+\frac{1}{2 \Delta} \frac{\mathrm{d} \Delta}{\mathrm{d} t} J \sin (2 \zeta+2 t) .
$$

Then we average the Hamiltonian over fast oscillations with the frequency of order unity. Neglecting the terms of order $\epsilon^{2}$ and higher, we obtain the averaged Hamiltonian as follows:

$$
\bar{H}(\bar{\zeta}, \bar{J}, \bar{t})=\bar{J}\left[\frac{\lambda}{2}+C(\bar{t}) \sin (2 \bar{\zeta})+D(\bar{t}) \cos (2 \bar{\zeta})\right]
$$

where $\bar{t}=t-\tilde{k}_{X} \phi_{k}, \bar{J}=J+\mathcal{O}(v \epsilon)$,

$$
\begin{aligned}
C(\bar{t}) & =X_{1}(\bar{t}) \sin \left(v \bar{t}-\mu_{1} \bar{\alpha}\right)-X_{2}(\bar{t}) \sin \left(v \bar{t}+\sigma \mu_{2} \bar{\alpha}\right) \\
& +Y_{1}(\bar{t}) \cos \left(v \bar{t}-\mu_{1} \bar{\alpha}\right)+Y_{2}(\bar{t}) \cos \left(v \bar{t}+\sigma \mu_{2} \bar{\alpha}\right),
\end{aligned}
$$




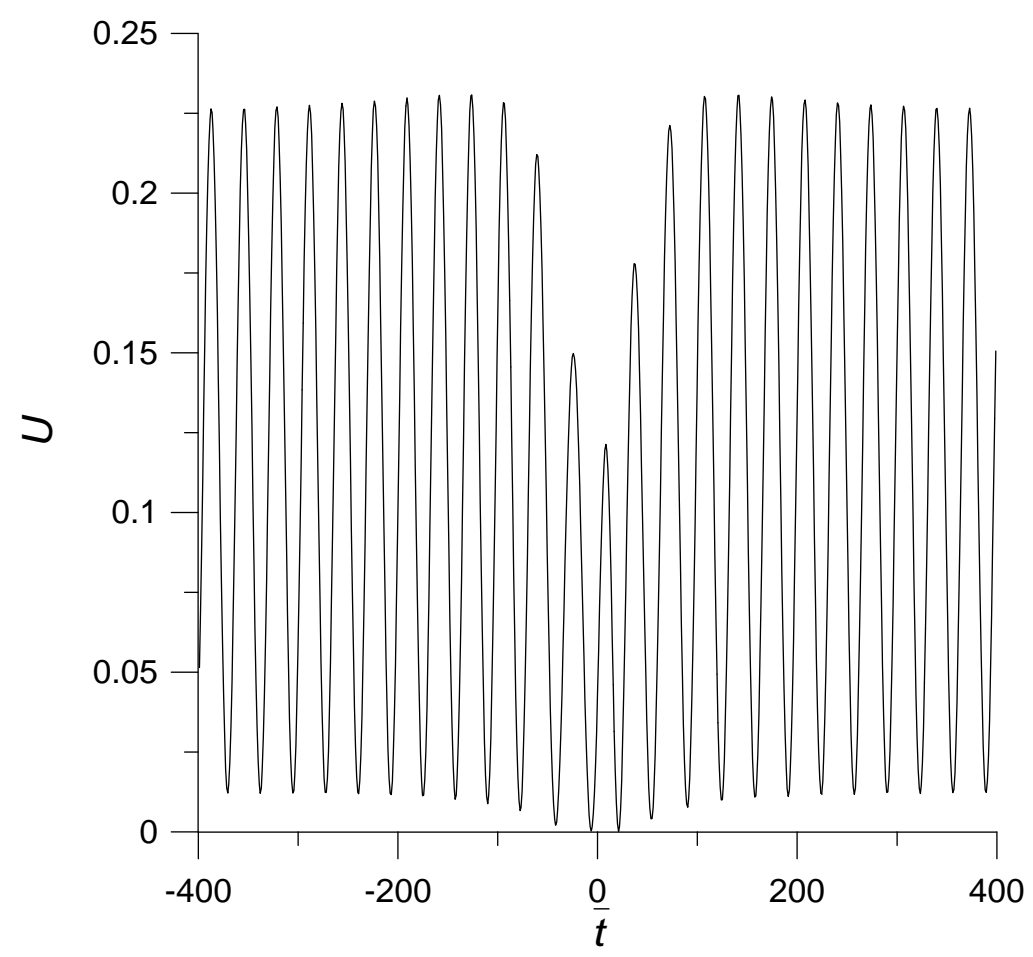

Figure 6. Potential (56) for the gaps $\left(\mu_{1}, \nu_{1}\right)=(2,1)$ and $\left(\mu_{2}, \nu_{2}\right)=(3,1)$ in the W7-AS shot No. 54937 at $r / a=0.45(\bar{s} \approx 0.016, v \approx 0.095)$.

$$
\begin{aligned}
D(\bar{t})= & -X_{1}(\bar{t}) \cos \left(v \bar{t}-\mu_{1} \bar{\alpha}\right)-X_{2}(\bar{t}) \cos \left(v \bar{t}+\sigma \mu_{2} \bar{\alpha}\right) \\
& +Y_{1}(\bar{t}) \sin \left(v \bar{t}-\mu_{1} \bar{\alpha}\right)-Y_{2}(\bar{t}) \sin \left(v \bar{t}+\sigma \mu_{2} \bar{\alpha}\right) \\
\bar{\zeta}=\zeta- & \frac{v \phi_{k}}{2} \frac{\mu_{1}+\sigma \mu_{2}}{\mu_{1}-\sigma \mu_{2}}+\mathcal{O}(v \epsilon), \quad \bar{\alpha}=\alpha-\frac{2 v \phi_{k}}{\mu_{1}-\sigma \mu_{2}}+\mathcal{O}(v \epsilon) .
\end{aligned}
$$

The functions $X_{j}(\bar{t})$ and $Y_{j}(\bar{t})$ for $j=1,2$ are given by

$$
X_{j}(\bar{t})=\frac{\epsilon_{j}^{\theta \theta}+\epsilon_{j}^{\psi \psi} \bar{s}^{2} \bar{t}^{2}}{1+\bar{s}^{2} \bar{t}^{2}}, \quad Y_{j}(\bar{t})=\frac{2 \epsilon_{g j}^{\psi \theta} \bar{s} \bar{t}}{1+\bar{s}^{2} \bar{t}^{2}},
$$

where $\epsilon_{j}^{\psi \psi}=\epsilon_{g j}^{\psi \psi}-2 \epsilon_{B} \approx\left(\epsilon_{g j}^{\psi \psi}+\epsilon_{c j}^{\psi \psi}\right) / 2, \epsilon_{j}^{\theta \theta}=\epsilon_{g j}^{\theta \theta}-2 \epsilon_{B}$. We observe that there is no parameter corresponding to $\phi_{k}$ after the averaging (one can expect that the dependence of $\lambda$ on $\phi_{k}$ is very weak, $\sim \exp \left(-c \bar{s}^{-1}\right)$ with $c \sim 1$, which is how averaged solutions typically depend on the phase of fast oscillations).

Acting like in subsection 2.4, we reduce the Hamiltonian system (49) to a Schrödinger equation. Here we will restrict ourselves to the case of $\bar{s} \ll \epsilon$, when the calculations are less cumbersome. Then, introducing new variables $(\bar{\Phi}, \bar{P})$ with the canonical transformation

$$
\bar{\Phi}=(2 \bar{J})^{1 / 2} \cos (\bar{\zeta}), \quad \bar{P}=(2 \bar{J})^{1 / 2} \cos (\bar{\zeta}),
$$

and excluding $\bar{P}$ from Hamilton's equations, we obtain

$$
\frac{d^{2} \bar{\Phi}}{d \bar{t}^{2}}+[E-U(\bar{t})] \bar{\Phi}=0,
$$


where $E=\lambda^{2} / 4$ and $U(\bar{t})=C^{2}+D^{2}$. One can present the effective potential $U$ in the form

$$
\begin{aligned}
U=2\left[X_{1}^{2}(\bar{t})\right. & \left.+Y_{1}^{2}(\bar{t})\right]^{1 / 2}\left[X_{2}^{2}(\bar{t})+Y_{2}^{2}(\bar{t})\right]^{1 / 2} \cos \left[2 v \bar{t}+\left(\sigma \mu_{2}-\mu_{1}\right) \bar{\alpha}+\gamma(\bar{t})\right] \\
& +X_{1}^{2}(\bar{t})+X_{2}^{2}(\bar{t})+Y_{1}^{2}(\bar{t})+Y_{2}^{2}(\bar{t}),
\end{aligned}
$$

where

$$
\gamma=\tan ^{-1} \frac{X_{2}(\bar{t}) Y_{1}(\bar{t})-X_{1}(\bar{t}) Y_{2}(\bar{t})}{X_{1}(\bar{t}) X_{2}(\bar{t})+Y_{1}(\bar{t}) Y_{2}(\bar{t})}
$$

and the branch of $\tan ^{-1}$ is chosen so that $\gamma$ is continuous and $\gamma(0)=0$. A typical example of the behaviour of the potential (56) is given in figure 6, where $U$ is calculated for the widest continuum gaps, $\left(\mu_{1}, \nu_{1}\right)=(2,1)$ and $\left(\mu_{2}, \nu_{2}\right)=(3,1)$, in a magnetic configuration of W7-AS. Like in subsection 2.4, the oscillations of the potential result from beatings of the two equilibrium harmonics. As a result, it has numerous wells, each possessing multiple bounded states. As mentioned above, equation (43) turns into the continuum equation at $\phi \rightarrow \pm \infty$. As a result, the potential $U$ is approximately periodic at large $\phi$. The frequency levels in far potential wells spread into continuum bands due to tunnelling between the wells, as was described in the previous section, the corresponding wave functions remaining finite at $\phi \rightarrow \pm \infty$. In contrast to that, the bounded states of the central wells, where the potential differs from the potential of the far wells by more than the characteristic continuum band width, are restricted to one well (at most, to several wells). They vanish at $\phi \rightarrow \pm \infty$ and represent eigenmodes of the discrete spectrum. It follows from equations (40) and (56) that the central wells (for which $\gamma \approx 0$ ) are located at the same places where the continuum waves are trapped (provided that $\left.\operatorname{sgn}\left(\epsilon_{1}^{\psi \psi} \epsilon_{2}^{\psi \psi}\right)=\operatorname{sgn}\left(\epsilon_{1}^{\theta \theta} \epsilon_{2}^{\theta \theta}\right)\right)$. Thus, our assumption that the AEs are localized in waveguides of the same shape as the continuum waves is confirmed. In particular, the HAE modes produced by the dominant Fourier harmonics with $(\mu, \nu)=(2,1)$ and $(3,1)$ are typically localized at the inner circumference of the torus, which is determined by the signs of the corresponding harmonics of the configuration. As mentioned above, these two harmonics are to a large extent responsible for all $\mathrm{AC}$ gaps with $\nu=1$. Hence, this conclusion may be true also for the mirror-induced Alfvén eigenmodes (MAE).

Note that the nature of this "anti-ballooning" structure of the considered HAEs differs from the nature of the anti-ballooning structure of the odd TAE. The latter results from the approximate compensation of the two principal harmonics of the mode at $\theta=0$. In contrast to this, the considered HAEs are poloidally inhomogeneous because they are evanescent near the outer circumference of the torus. This implies that the mode consists of many Fourier harmonics (much more than two) and it is much stronger localized poloidally.

\section{Experimental observation of high-frequency Alfvén eigenmodes}

At the final stage of W7-AS shot \# 54937, high-frequency activity of plasma was observed. The instabilities were detected by Mirnov coils, which included two poloidal 
Probe Positions: MIR-3, $\phi=129^{\circ}$

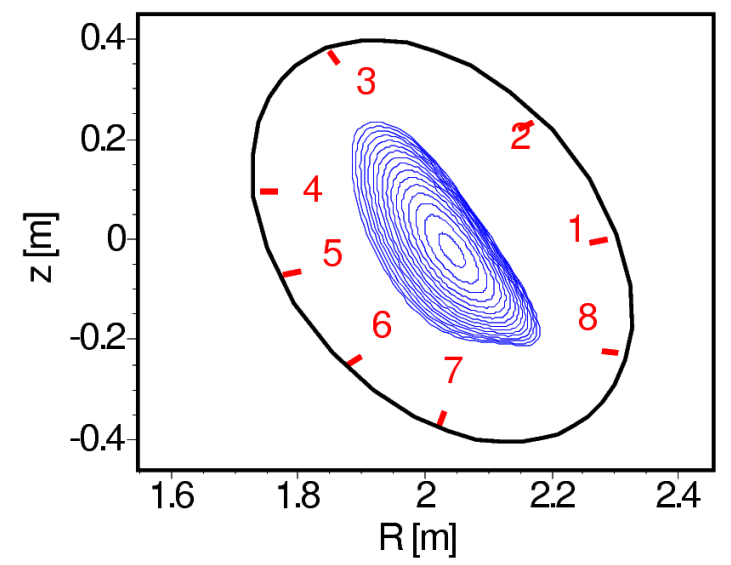

Probe Positions: MIR-5, $\phi=303.5^{\circ}$

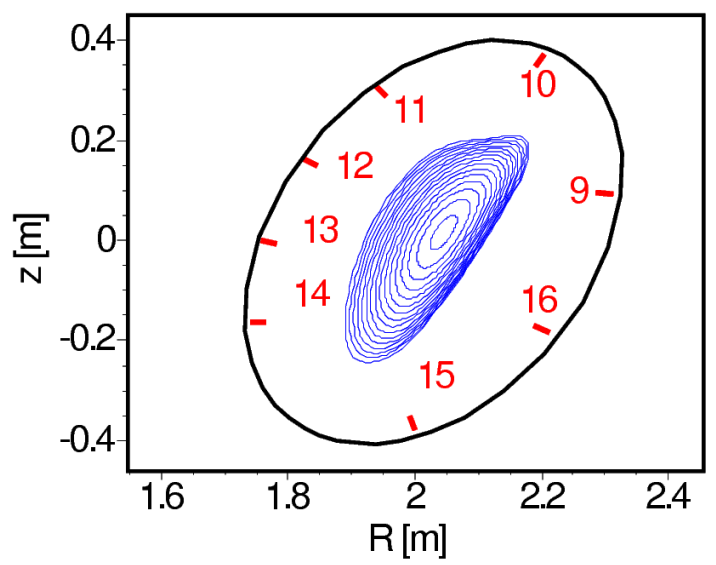

Figure 7. Locations of Mirnov coils.
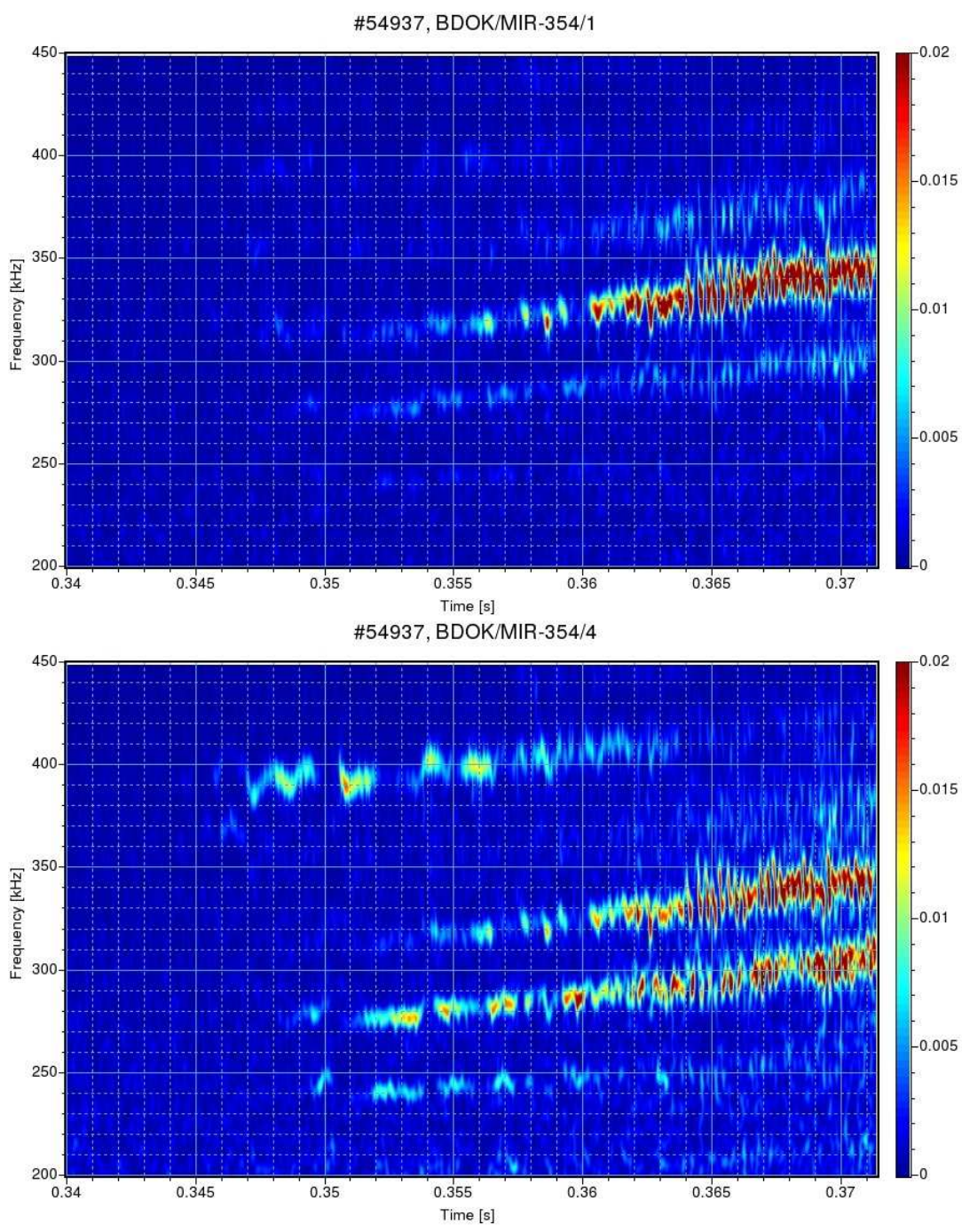

Figure 8. Frequency spectrum of Mirnov signals in W7-AS shot \# 54937 vs time. Upper panel, coil 1; lower panel, coil 4. 

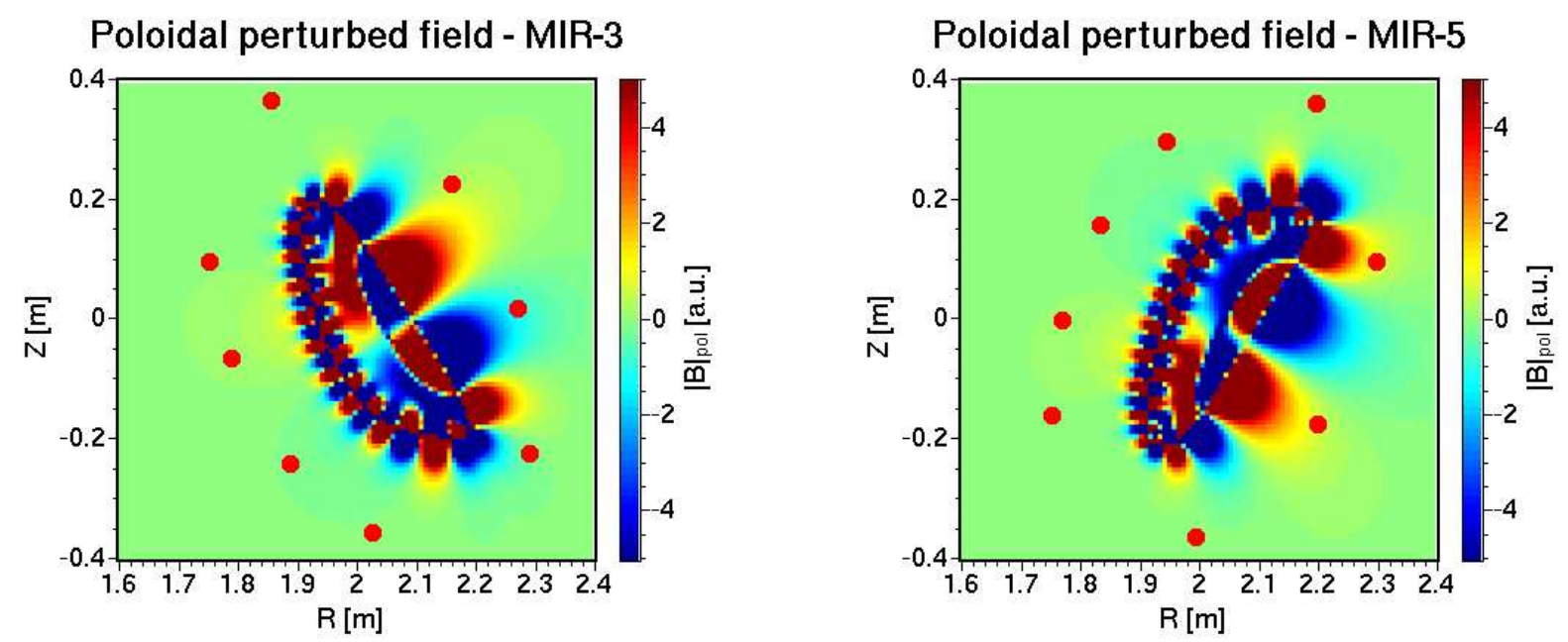

Figure 9. Spatial distributions of the poloidal component of the perturbed magnetic field - solid red dots show the probe positions. Left: $\phi=129^{\circ}$; right: $\phi=303.5^{\circ}$.

arrays, MIR-3 and MIR-5, with a sample rate of $1 \mathrm{MHz}$ [39]. The arrays were located at $\phi=129^{\circ}$ and $\phi=303.5^{\circ}$, respectively (see figure 7 ). A Lomb periodogram analysis of the Mirnov coil signals was carried out as described in [39]. The obtained spectrum of the activity consisted of several lines in the range of $200-450 \mathrm{kHz}$ (see figure 8), which well exceeded the frequencies of TAE and EAE modes (less than $100 \mathrm{kHz}$ in the plasma core). Each line seems to be produced by a separate instability since the amplitudes of the lines evolved independently. The identification of the instabilities as Alfvén eigenmodes is supported by the fact that their frequencies slowly increased with a decrease of the plasma density. In fact, each line consisted of separate bursts with the duration $\Delta t \sim 0.2 \mathrm{~ms}$. The frequency width of each burst was $\Delta f \sim 10 \mathrm{kHz}$. As $\Delta f \Delta t \sim 2$, the observed frequency width may be completely caused by the finite duration of the burst, although weak frequency chirping is not excluded.

Comparison of the frequency spectra of the activity at different Mirnov coils reveals that the spectral lines exhibit different dependence on $\theta$. Figure 8 shows the spectra of the signals on the coils 1 and 4 located on outer and inner circumferences of the torus, respectively. The comparison of the two spectra shows that most spectral lines (the lines with the frequencies about 250,290 and $410 \mathrm{kHz}$ at $t=0.36 \mathrm{~s}$ ) are much stronger at the coil 4, whereas the $320 \mathrm{kHz}$ line has approximately equal amplitudes at both coils. Thus, the Mirnov signals exhibit strong poloidal inhomogeneity, which is different for different spectral lines, and some spectral lines are much stronger at the inner circumference of the device.

To answer the question if this inhomogeneity reflects a real variation of the wave amplitude within the plasma, one needs to take account of geometrical effects (the poloidal variations of both $|\nabla \theta|$ and the distance between the plasma and the coils). These effects can also result in poloidal variations of the signal amplitudes, as demonstrated in figure 9, which shows a simulation of the poloidal magnetic field of a perturbation with $m=13$ in the vacuum region. The perturbation is modelled by a 

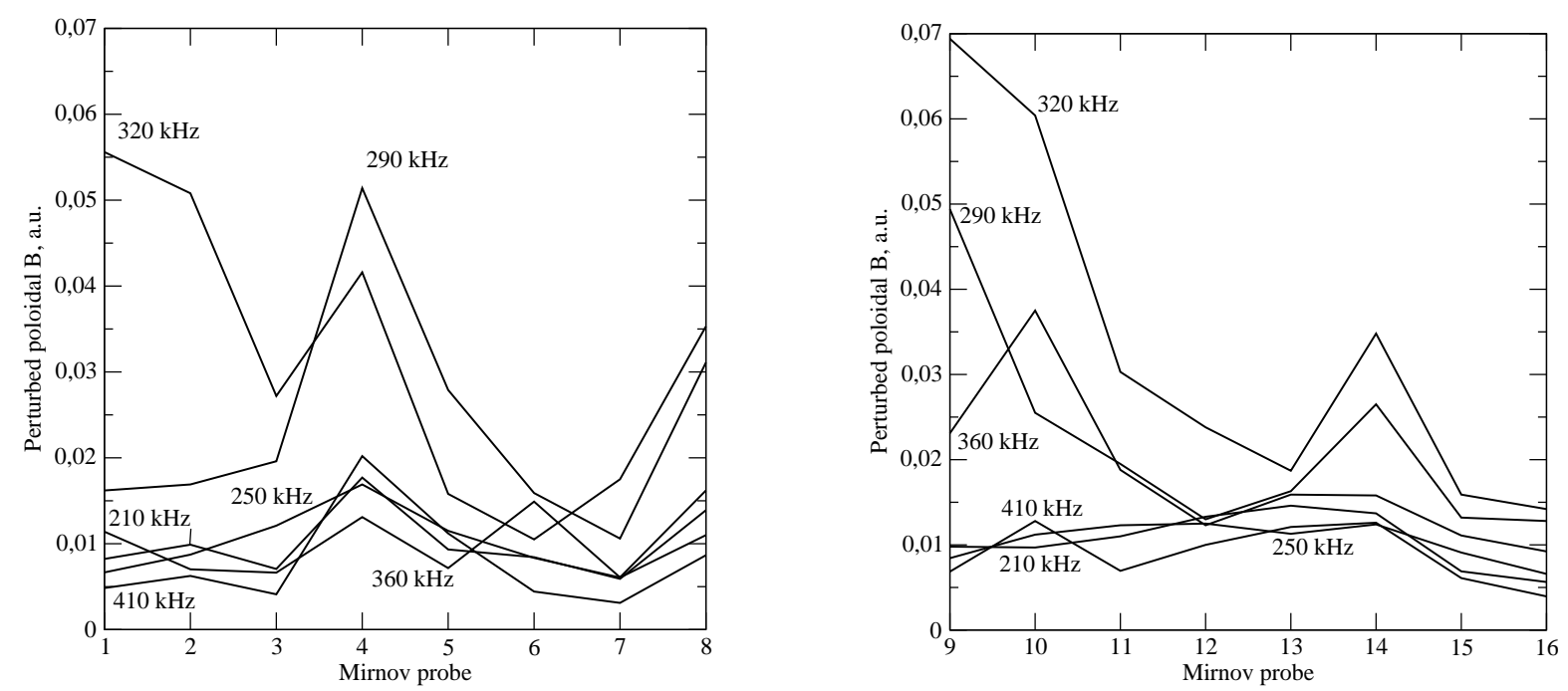

Figure 10. The maximum amplitudes of the high-frequency spectral lines on Mirnov probes. The frequencies of the lines are given for $t=0.36 \mathrm{~s}$. Left, array MIR-3. Right, array MIR-5.

surface current with the density $\propto \cos (m \theta-n \phi-\omega t)$. One can see that the perturbation field near the plasma is stronger at the inner circumference, which is explained by higher $|\nabla \theta|$ resulting in higher current density. However, the smaller poloidal wave length at the inner circumference leads to faster decay of the wave in the vacuum region. As a result, the perturbation field on the coils is much stronger at the outer circumference of the torus in figure 9 . For small $m$, the decay in the vacuum region is not strong, and the field at the coils is stronger at the inner circumference. Although the wave propagation in the vacuum region is, in reality, more complicated than in this model, and surface currents are not a perfect substitute for a MHD-mode, it seems that the geometrical effects described above indeed take place.

However, the geometrical effects discussed above cannot completely account for the observed dependence of the signal amplitude on the coil position. Figure 10 shows the maximum amplitudes of the spectral lines on all Mirnov probes of the two arrays. One can see that the perturbation is weak (for all spectral lines) at the coils 6 and 7 of the MIR-3 array, which are located at the inner circumference. At the same time, the perturbation is weak at the coils 15 and 16 of the MIR-5 array, which are located at the outer circumference. If one takes into account that the arrays are positioned almost symmetrically in the toroidal angle $\left(129^{\circ}=2 \times 360^{\circ} / N-15^{\circ}\right.$ and $\left.303.5^{\circ}=4 \times 360^{\circ} / N+15.5^{\circ}\right)$, the difference in the poloidal distribution at the two arrays seems, at a first glance, to contradict the stellarator symmetry (note that amplitude of any perturbation, the harmonics of which are coupled via periodic equilibrium quantities, can be shown to obey the stellarator symmetry). As the Mirnov coils had been calibrated, a strong difference in the coil sensitivity could not be an explanation. In principle, the stellarator symmetry of the mode amplitude could be broken for an EPM (energetic particle mode) if the distribution function of the fast particles exciting 
the mode is asymmetric (different in different field periods). However, it is hard to expect strong aperiodicity of the fast ion distribution since the ions are produced by tangential NBI (neutral beam injection), so that they are predominantly passing. A possible explanation is that the observed amplitude of the perturbations on the wall rapidly oscillates in the poloidal direction. The signal amplitudes shown in figure 10 support this explanation: The amplitudes on neighboring probes can be very different, and, moreover, there is some obvious correlation between the intensities of all spectral lines (see, e.g, the probes 4, 5 and 6). Because the poloidal positions of the probes are different in the two arrays, such oscillations can produce a strong difference between the poloidal distributions of the wave amplitude. The causes of such oscillations are not quite clear, but they might include variations of the angle between the coils and the wave polarization, which might be enhanced by field corrugations. Such corrugations were typically observed in discharges with high $\iota>0.5(\iota=0.55-0.58$ at $t=0.36 \mathrm{~s}$ in the discussed shot).

Thus, the poloidal dependence of the wave amplitude is obscured by rapid oscillations, the nature of which is not completely understood. Nevertheless, some spectral lines show clear signs of ballooning $(320 \mathrm{kHz})$ or anti-ballooning $(210,250$, and $410 \mathrm{kHz}$ ) behaviour. As shown above, due to geometrical effects the wave amplitude on the probes may exhibit ballooning or anti-ballooning behaviour even if the wave amplitude at the plasma boundary does not depend on $\theta$. Since the geometrical effects depend on $m$, we attempted to find $m$ of the observed modes, using the Lomb periodogram analysis described in [39]. The attempt was not quite successful. We failed to find perturbation harmonics, for which the continuum branches have the same frequency as the observed instabilities. However, $k_{\|} v_{A}$ was not far from the observed frequencies for some harmonics having $m \sim 10-15$. As follows from figure 9 , the observed signals for such $m$ must exhibit clear ballooning behaviour. Then the antiballooning poloidal dependence of the signals with the frequencies of 210, 250, and $410 \mathrm{kHz}$ cannot be attributed to geometrical effects and seems to reflect a variation of the instability amplitude in the plasma.

We used the AC code COBRA to calculate the $\mathrm{AC}$ at $t=0.36 \mathrm{~s}$. The results are shown in figure 11, where the frequency gaps in the continuum are labelled by the corresponding coupling numbers $(\mu, \nu)$. The frequency range of interest corresponds to helicity-induced $(\mu \neq 0, \nu \neq 0)$ and mirror-induced $(\mu=0, \nu=1)$ gaps. In particular, the $210 \mathrm{kHz}$ and $250 \mathrm{kHz}$ instabilities are in $(2,1)$-gap (if they are excited in the plasma core), whereas the $410 \mathrm{kHz}$ instability lies coincides with the $(-2,1)$-gap. The latter arises as a combination gap (the $(-2,1)$ harmonics of the relevant equilibrium quantities are too small) and results from joint action of other helical harmonics, the largest of which are the harmonics with $(\mu, \nu)=(2,1)$ and $(3,1)$. We conclude that the observed anti-ballooning behaviour of some instabilities agrees well with the theoretical results presented in section 3, which predict the anti-ballooning localization of the modes produced by the helical harmonics. The $320 \mathrm{kHz}$ instability shows a ballooning dependence on $\theta$, which can be attributed to geometrical effects. It lies in a spectral 


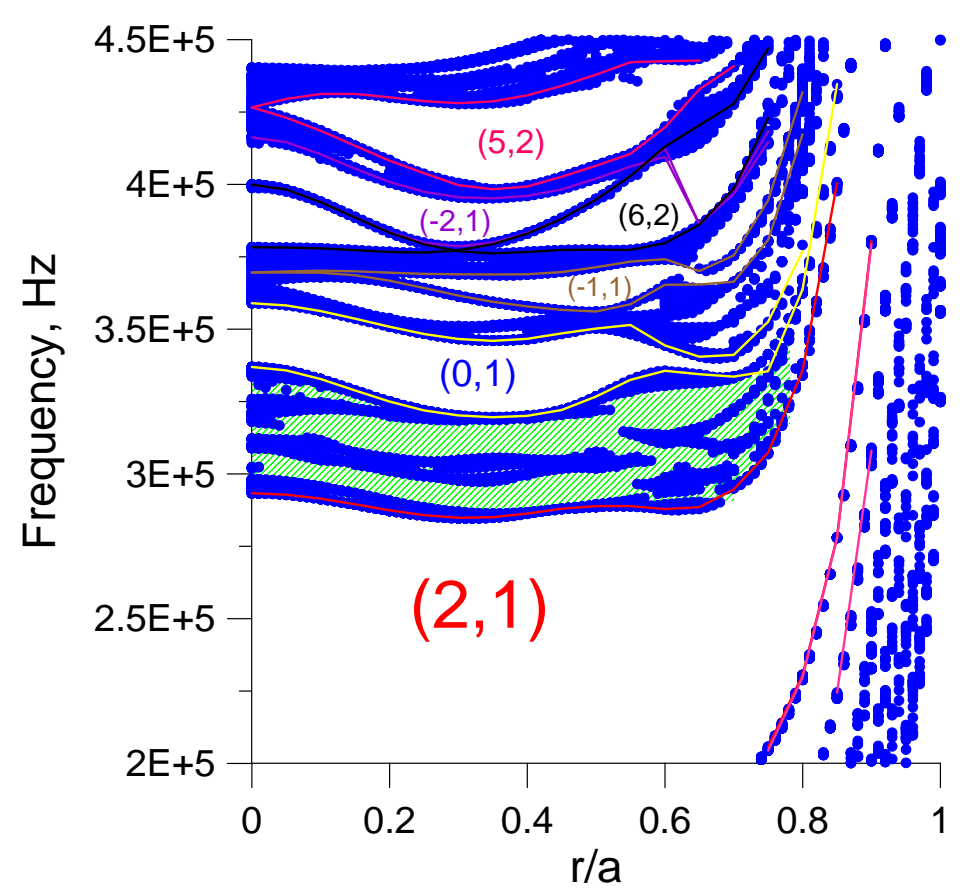

Figure 11. The Alfvén continuum in W7-AS shot \# 54937. Dots, the continuum; the gaps are labelled with the corresponding numbers $(\mu, \nu)$. The region where the calculations are not reliable is hatched.

range of strong interaction of gaps with different values of $\nu$ (e.g., the $(1,1)$ - and $(7,0)$ gaps). Our calculations of the continuum in this range were not successful because of the extremely complicated Fourier structure of continuum wave functions. This means that the waveguides of this eigenmode could have a more complicated shape, which would explain the absence of observable poloidal trapping.

Thus, the experimental observations indicate that some high-frequency instabilities in W7-AS are trapped at the inner circumference of the plasma torus, as predicted by our theory, although the observations are obscured by some factors causing rapid poloidal oscillations of the observed wave amplitude.

\section{Conclusions}

We have found that the interaction of two Fourier harmonics of the plasma equilibrium can result in trapping of the wave functions of the Alfvén continuum in certain "waveguides". The trapping occurs when the periods of the two harmonics along the field lines are sufficiently close in comparison with the harmonic amplitudes (see equation (39)) and is explained by the interference of the harmonics, which add at one places and cancel at other ones. When the periods of the two harmonics exactly coincide (which means that the continuum gaps produced by these harmonics cross), the wave functions are localized at single field lines. This is shown to lead to "annihilation" of the continuum gaps: the width of the joint gap at the crossing point is equal to the difference of the widths of separate gaps. 
The trapping is typical in the high-frequency part of the Alfvén continuum (the range of the helicity- and mirror-induced gaps), where the gaps (at least, in stellarators with high $N$ ) are close enough. In the typical case when this part of the spectrum is dominated by the harmonics $(2,1)$ and $(3,1)$ (the helical elongation and triangularity), the waves are trapped at the inner circumference of the flux surface. In addition, the trapping takes places near crossings of continuum gaps (one can expect that crossings of major gaps are usual in low- $N$ devices, like NCSX). Then the structure of the wave guides is more complicated.

It is known that the oscillations of the continuous spectrum, in contrast to eigenmodes, cannot be excited in practice since they are strongly damped (unless the drive overcomes the damping, as occurs for the EPMs). Nevertheless, a change of the structure of the continuum oscillations is important from practical point of view. The trapping may affect the energy absorption of Alfvén waves since the angular structure of an Alfvén wave is known to become similar to that of the continuum wave function when the wave approaches the local resonance with the continuum [29]. In addition, it is known that the structure of the Alfvén eigenmodes is closely associated with the structure of the continuum. Indeed, we have shown that the eigenmodes produced by two equilibrium harmonics with close periods are trapped in waveguides of the same shape as the continuum wave functions. In particular, this means the highfrequency eigenmodes produced by the $(2,1)$ - and $(3,1)$-harmonics typically exhibit "anti-ballooning" behaviour (i.e., they are trapped at the inner circumference of the plasma torus), which is determined by the signs of the corresponding harmonics of the metric tensor.

This prediction of our theory is compared with experimental observations made in a W7-AS shot, in which high-frequency Alfvénic activity was observed. Although the poloidal dependence of the wave amplitude was obscured by some factors (presumably, by corrugations of the flux surfaces, which resulted in variations of the angle between the wave polarization and the Mirnov coils), there are indications that anti-ballooning behaviour of some instabilities was indeed observed.

\section{Acknowledgments}

The research described in this publication was made possible in part by the Partner Project Agreement No. P-034f between the Science and Technology Center in Ukraine, the Institute for Nuclear Research, Ukraine, and the Max-Planck-Institut für Plasmaphysik, Germany. One of the authors (Y.Y.) acknowledges the hospitality of the Max-Planck-Institut für Plasmaphysik.

\section{References}

[1] Wong K-L 1999 Plasma Phys. Control. Fusion 41 R1

[2] Weller A, Anton M, Geiger J, Hirsch M, Jaenicke R, Werner A, W7-AS Team, Nührenberg C, Sallander E and Spong D A 2001 Phys. Plasmas 8931 
[3] Toi K et al 2000 Nucl. Fusion 401349

[4] Kolesnichenko Ya I, Yakovenko Yu V, Weller A, Werner A, Geiger J, Lutsenko V V and Zegenhagen S 2005 Phys. Rev. Lett. 94165004

[5] Goedbloed J P, Holties H A, Poedts S, Huysmans G T A and Kerner W 1993 Plasma Phys. Control. Fusion 35 B277

[6] Kramer G J et al 2000 Nucl. Fusion 401383

[7] Kolesnichenko Ya I, Lutsenko V V, Wobig H and Yakovenko Yu V 2002 Phys. Plasmas 9517

[8] Grossman W and Tataronis J 1973 Z. Phys. 261217

[9] Appert K, Gruber R, Troyon F and Vaclavik J 1982 Plasma Phys. 241147

[10] Ross D W, Chen G L and Mahajan S M 1982 Phys. Fluids 25652

[11] Kieras C E and Tataronis J A 1982 J. Plasma Phys. 28395

[12] Cheng C Z and Chance M S 1986 Phys. Fluids 293695

[13] Betti J R and Freidberg J P 1992 Phys. Fluids B 41465

[14] Mett R R and Mahajan S M 1992 Phys. Fluids B 42885

[15] Heeter R F, Fasoli A, Borba D, Start D, Ali-Arshad S and Fisch N 1997 5th IAEA Technical Committee Meeting on Alpha Particles in Fusion Research (Abingdon, 1997) (Abingdon: Joint European Torus) p 9

[16] Chen L 1994 Phys. Plasmas 11519

[17] Nakajima N, Cheng C Z and Okamoto M 1992 Phys. Fluids B 41115

[18] Nührenberg C 1999 Plasma Phys. Control. Fusion 411055

[19] Kolesnichenko Ya I, Lutsenko V V, Wobig H, Yakovenko Yu V and Fesenyuk O P 2000 Report IPP III/261, Max-Planck-Institut für Plasmaphysik (Garching bei München, 2000) Phys. Plasmas 20018491

[20] Nührenberg C 2000 ISSP-19 "Piero Caldirola", Theory of Fusion Plasmas, ed J W Connor, O Sauter and E Sindoni (Bologna: Editrice Compositori - Società Italiana di Fisica) p 313

[21] Salat A 1992 Plasma Phys. Control. Fusion 341339

[22] Yakovenko Yu V et al 2004 Proc. 20th IAEA Fusion Energy Conf. (Vilamoura, November 2004) (Vienna: IAEA), CD-ROM file IAEA-CN-116/TH/P4-48

[23] Yakovenko Yu V, Kolesnichenko Ya I, Fesenyuk O P, Weller A, Zegenhagen S, Werner A and Geiger J 2006 Proc. 13th Int. Congr. on Plasma Phys. (Kiev, May 2006) (Kiev: Boholyubov Institute of Theoretical Physics), CD-ROM file B022p

[24] Zegenhagen S, Yakovenko Yu V, Kolesnichenko Ya I, Werner A, Geiger J, Weller A and Fesenyuk O P 2006 Proc. 33th EPS Conf. on Plasma Phys. (Rome, June 2006) (ECA) P2.117, to be published

[25] Kolesnichenko Ya I, Lutsenko V V, Marchenko V S, Weller A, White R B, Yakovenko Yu V and Yamazaki K 2007 Magnetohydrodynamic activity and energetic ions in fusion plasmas Plasma Phys. Control. Fusion 49, to be published

[26] Dewar R L and Glasser A H 1983 Phys. Fluids 263038

[27] Wagner F et al 2005 Phys. Plasmas 12072509

[28] Boozer A H 1981 Phys. Fluids 241999

[29] Salat A and Tataronis J A 2001 Phys. Plasmas 81207

[30] Simon B 1982 Adv. Appl. Math. 3463

[31] Fujiwara M et al 2001 Nucl. Fusion 411355

[32] Kolesnichenko Ya I, Yamamoto S, Yamazaki K, Lutsenko V V, Nakajima N, Narushima Y, Toi K and Yakovenko Yu V 2004 Phys. Plasmas 11158

[33] Moler C and Stewart G W 1973 SIAM J. Num. Anal. 10241

[34] Fesenyuk O P, Kolesnichenko Ya I, Lutsenko V V, White R B and Yakovenko Yu V 2004 Phys. Plasmas 115444

[35] Littlejohn R G 1982 J. Math. Phys. 23742

[36] Candy J, Breizman B N, Van Dam J W and Ozeki T 1996 Phys. Letters A 215299

[37] Dewar R L, Chance M S, Glasser A H, Greene J M and Frieman E A 1979 Report PPPL-1587, 
Princeton Plasma Physics Laboratory (Princeton: Princeton University)

[38] Zonca F and Chen L 1993 Phys. Fluids B 53668

[39] Zegenhagen S, Werner A, Weller A and Klinger T 2006 Plasma Phys. Control. Fusion 481333 